See discussions, stats, and author profiles for this publication at: https://www.researchgate.net/publication/325492505

\title{
Level Set Regularization Using Geometric Flows
}

Article in SIAM Journal on Imaging Sciences · January 2018

DOI: $10.1137 / 17 \mathrm{M} 1139722$

CITATIONS

0

4 authors:

Luis Alvarez

Universidad de Las Palmas de Gran Canaria

160 PUBLICATIONS 4,131 CITATIONS

SEE PROFILE

Jesus Ildefonso Diaz

Complutense University of Madric

546 PUBLICATIONS 4,503 CITATIONS

SEE PROFILE

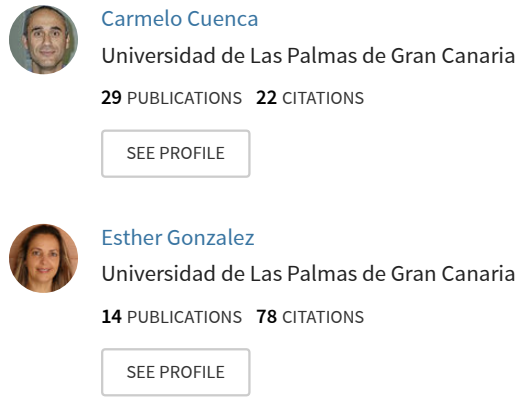

Some of the authors of this publication are also working on these related projects:

Educative Games View project

Modelización matemática de los procesos de calibración de cámaras de vídeo (MTM2010-17615) View project 


\title{
Level Set Regularization Using Geometric Flows*
}

\author{
Luis Alvarez $^{\dagger}$, Carmelo Cuenca ${ }^{\dagger}$, Jesús Ildefonso Díaz ${ }^{\ddagger}$, and Esther González ${ }^{\dagger}$
}

\begin{abstract}
In this paper we study a geometric partial differential equation including a forcing term. This equation defines a hypersurface evolution that we use for level set regularization. We study the shape of the radial solutions of the equation and some qualitative properties about the level set propagations. We show that under a suitable choice of the forcing term, the geometric equation has nontrivial asymptotic states and it represents a model for level set regularization. We show that by using a forcing term which is merely a bounded Hölder continuous function, we can obtain finite time stabilization of the solutions. We introduce an explicit finite difference scheme to compute numerically the solution of the equation and we present some applications of the model to nonlinear two-dimensional image filtering and three-dimensional segmentation in the context of medical imaging.
\end{abstract}

Key words. geometric flows, level sets evolution, partial differential equations

AMS subject classifications. 35K55, 35D40, 35R37

DOI. $10.1137 / 17 \mathrm{M} 1139722$

1. Introduction. In this paper we consider the Cauchy problem for the parabolic perturbed mean curvature type equation

$$
\left\{\begin{array}{llc}
\frac{\partial u}{\partial t}=F\left(\nabla u, \nabla^{2} u\right)+k(x)|\nabla u| & \text { in } & (0, T) \times \mathbf{R}^{n} \\
u(0, x)=u_{0}(x) & \text { on } & \mathbf{R}^{n}
\end{array}\right.
$$

where $\nabla u$ and $\nabla^{2} u$ denote respectively the gradient and the Hessian of $u$ in spatial variables, $k: \mathbf{R}^{n} \rightarrow \mathbf{R}$ is a bounded Hölder continuous function which introduces a forcing term in (1), and $F: \mathbf{R}^{n}-\{0\} \times \mathbf{S}^{n} \rightarrow \mathbf{R}$ is a given continuous function satisfying

$$
\begin{gathered}
-F(p, X) \leq-F(p, Y) \quad \forall X, Y \in \mathbf{S}^{n} \text { with } X \geq Y, \forall p \in \mathbf{R}^{n}, \\
F(\lambda p, \lambda X+\sigma p \otimes p)=\lambda F(p, X) \quad \forall \lambda>0, \sigma \in \mathbf{R}, \forall p \in \mathbf{R}^{n}, \forall X \in \mathbf{S}^{n},
\end{gathered}
$$

where $\otimes$ denotes the tensor product in $\mathbf{R}^{n}$. Here $\mathbf{S}^{n}$ denotes the space of the $n \times n$ real and symmetric matrices with the usual Loewner order. We say (1) generates a geometric flow if $F$ satisfies (3) (we point out that as $k|\lambda p|=\lambda k|p|$, the forcing term does not change the

\footnotetext{
${ }^{*}$ Received by the editors July 21, 2017; accepted for publication (in revised form) April 11, 2018; published electronically May 31, 2018.

http://www.siam.org/journals/siims/11-2/M113972.html

Funding: This research was partially supported by the MINECO projects MTM2016-75339-P and MTM201457113-P (AEI/FEDER, UE), the DGISPI (Spain), and the Research Group MOMAT (910480) supported by the Universidad Complutense de Madrid.

${ }^{\dagger}$ CTIM (Centro de Tecnologías de la Imagen), Departamento de Informática y Sistemas, Universidad de Las Palmas de Gran Canaria, 35017 Las Palmas de G.C., Spain (lalvarez@ulpgc.es, carmelo.cuenca@ulpgc.es, esther. gonzalez@ulpgc.es).

${ }^{\ddagger}$ Instituto de Matemática Interdisciplinar, Departamento de Matemática Aplicada, Universidad Complutense de Madrid, 28040-Madrid, Spain (jidiaz@ucm.es).
} 
geometric character of (3)). In this paper, we pay special attention to the mean curvature operator given by

$$
F\left(\nabla u, \nabla^{2} u\right)=\operatorname{div}\left(\frac{\nabla u}{|\nabla u|}\right)|\nabla u|=\sum_{i=1}^{n} u_{x_{i} x_{i}}-\sum_{i, j=1}^{n} \frac{u_{x_{i}} u_{x_{j}}}{|\nabla u|^{2}} u_{x_{i} x_{j}}
$$

which satisfies (2)-(3). One of the main interests of the geometric flows in the context of computer vision is that they are closely related to the following level set formulation of front propagations introduced in [27], [30]: given a hypersurface $\Gamma_{0} \subset \mathbf{R}^{n}$, we embed $\Gamma_{0}$ in a function $u_{0}: \mathbf{R}^{n} \rightarrow \mathbf{R}$ in such a way that $\Gamma_{0}=\partial\left\{x: u_{0}(x)<0\right\}$, and we make $u_{0}$ evolve using the partial differential equation

$$
\frac{\partial u}{\partial t}+F_{\text {ext }}|\nabla u|=0
$$

The hypersurface evolution is then defined by the set $\Gamma_{t}=\partial\{x: u(t, x)<0\} . F_{\text {ext }}$ is a function representing the front propagation velocity in the hypersurface normal direction. This elegant formulation has been extensively applied in the literature to address a variety of problems. For instance, in [10], the authors propose an active contour model where $F_{\text {ext }}$ is given by

$$
F_{\text {ext }}(x)=-g(x)\left(\operatorname{div}\left(\frac{\nabla u}{|\nabla u|}\right)(x)+\nu\right),
$$

where $\nu \in \mathbf{R}$ and $g(x) \geq 0$. In [13], [14] the authors introduce the "geodesic snake model" where $F_{\text {ext }}$ is given by

$$
F_{\text {ext }}(x)=-g(x)\left(\operatorname{div}\left(\frac{\nabla u}{|\nabla u|}\right)(x)+\nu\right)-\nabla g(x) \frac{\nabla u}{|\nabla u|}(x) .
$$

In the context of three-dimensional (3D) medical image segmentation, in [22], the authors propose a model for $3 \mathrm{D}$ vessel segmentation based on the front propagation velocity

$$
F_{\text {ext }}(x)=g(x)\left(1-\epsilon \kappa_{\min }(x)\right),
$$

where $\epsilon \in \mathbf{R}, g(x) \in[-1,1]$, and $\kappa_{\min }(x)$ represents the minimum value of the curvatures of the level set surface of $u$ passing by $x$.

We point out that if we choose the mean curvature operator (4) in (1), then (1) is equivalent to (5) with

$$
F_{\text {ext }}(x)=-\left(\operatorname{div}\left(\frac{\nabla u}{|\nabla u|}\right)(x)+k(x)\right) .
$$

From the above examples, we can observe that (5) is very flexible and that it can be adapted to many different problems according to the choice of the velocity term $F_{\text {ext }}$. From a mathematical point of view there are two main important issues related to the hypersurface evolution defined by (5). The first one is that the evolution of $\Gamma_{t}$ should be independent of the choice of the function $u_{0}$ where $\Gamma_{0}$ is embedded. The main interest of the geometric assumption 
(3) is that it ensures, as we will see below, that under the action of (1), $\Gamma_{t}$ is independent of the choice of $u_{0}$. The second one is whether (5) defines a well-posed problem, that is, we would like to show that (5) has a unique solution in some sense and that we have some other nice properties like the comparison principle, $L^{\infty}$ stability of the solutions, etc. The usual framework to study these issues is the viscosity solutions theory introduced at the end of the last century in some seminal papers, for instance, [17], [16], and [18] (see also the presentation made in the more recent monograph [20]). In the case of second order differential operators, the well-posedness of the problem is closely related to the ellipticity condition (2) and to the regularity of functions like $g(x)$ and $k(x)$ in the examples above. For instance, the existence and uniqueness of the viscosity solutions for (6), (7) with $g(x) \geq 0$ and $g, g^{\frac{1}{2}} \in W^{1, \infty}\left(\mathbf{R}^{n}\right)$ (for $n=2,3)$ have been shown in $[10,14,8]$. In most of the papers concerning the case in which a forcing term, $k(x)$, is involved, it is assumed that $k \in W^{1, \infty}\left(\mathbf{R}^{n}\right)$. In section 2 of this paper we study the case in which $k(x)$ is merely a bounded Hölder continuous function.

Equation (8) is a heuristic model that has not been well studied mathematically yet (notice that $g(x) \in[-1,1]$ and thus the associated differential operator loses its elliptic character). In fact, given the generality of (5), the possible absence of a correct mathematical treatment in terms of PDEs may justify many strange properties of the front propagation.

Comparison principle is a very important topic in PDE theory. In particular, in terms of front propagation, it means that if the evolution of two level sets $U_{t}=\{x: u(t, x)<0\}, \hat{U}_{t}=$ $\{x: \hat{u}(t, x)<0\}$ (where $u(t, x), \hat{u}(t, x)$ are solutions of the associated PDE) satisfying that $U_{0} \subseteq \hat{U}_{0}$, then $U_{t} \subseteq \hat{U}_{t} \forall t \geq 0$. This is a nice and useful behavior of the front propagation. For instance, in this paper, we make use of this comparison principle to compare the behavior of radial solutions with the one of the nonradial solutions of the equation. As we shall comment with more details in section 2 , the comparison principle for the viscosity solutions of the more general equation

$$
\frac{\partial u}{\partial t}+G\left(t, x, u, \nabla u, \nabla^{2} u\right)=0,
$$

proved in [21] under very general assumptions on $G$, can be applied to our mentioned framework. A characterization of the profile of the function $G$ using a "principle" approach including the geometric character of the equation was introduced in $[3,4,5]$.

As pointed out in [11] and [12], the topographic map, based on the image level set collection, is a robust and invariant image description suitable for image analysis. In terms of curve evolution, the geometric flows were studied in $[6,7,28,29]$ (see also the presentation made in the monograph [20]).

In this paper we focus our attention on the study of the evolution of the level sets under the action of the geometric flow (1). Our main motivation is that, as we will show in this paper, with a suitable choice of the forcing term $k(x),(1)$ can be used as a level set regularization model. Moreover (see, e.g., Lemma 8), if $k(x)$ is a non-Lipschitz function ( $k$ being merely Hölder continuous), then we can obtain finite time stabilization of the solutions.

The main contributions of the paper are (i) the qualitative study of the solutions of (1) with a forcing term $k(x)$ and specially the proof of some qualitative properties which are exclusive to the case in which $k(x)$ is merely a Hölder continuous function (based on the study of the level set propagation by using a sharp analysis of radial solutions), (ii) the proposal of (1) 
as a level set regularization model using an appropriate choice of the forcing term $k(x)$, and (iii) the illustration of the results of the proposed model in a variety of situations including nonlinear filtering of $2 \mathrm{D}$ images or $3 \mathrm{D}$ image segmentation in the context of medical imaging.

The rest of the paper is organized as follows. In section 2, we apply some previous results in the literature to get the existence and comparison of solutions of (1) in the context of the viscosity solution framework. In section 3, we study the hypersurface evolution obtained from the solutions of (1). Section 4 is devoted to the study of (1) in radial coordinates. In section 5 , we present some qualitative properties of the hypersurface evolution. An explicit finite difference discretization scheme for (1)-(4) is presented in section 6 , and some applications of (1)-(4) to image level set regularization including some experimental results are presented in section 7 . Finally, in section 8 we summarize the main conclusions of the paper.

2. On the existence and comparison of solutions. As we shall see in the next sections, one of the main qualitative properties of solutions of (1), i.e., the finite time stabilization of radial solutions, holds only when $k(x)$ is merely a Hölder continuous function (i.e., the property is not satisfied when $k(x)$ is assumed to be Lipschitz continuous). In most of the presentations on the existence and comparison of solutions of general formulations of the equation given in terms of (10) it is assumed that $G\left(t, x, u, \nabla u, \nabla^{2} u\right)$ is independent of $x$, or at least $G(t, x, u, p, O)$ is Lipschitz continuous on $x$. The motivation of this section is simply to recall the general results obtained in [21] and to check that they apply without any special difficulty to our framework: (1) with (9) and when $k(x)$ is a bounded Hölder continuous function. Notice that this corresponds to the choice

$$
G(t, x, u, p, X)=-\operatorname{trace}((I-\bar{p} \otimes \bar{p}) X)-k(x)|p|, \bar{p}:=p /|p|
$$

(see (4)). So we can write

$$
G(t, x, u, p, X)=-F(p, X)-k(x)|p| \quad \text { with } F(p, X):=\operatorname{trace}((I-\bar{p} \otimes \bar{p}) X) .
$$

We start by recalling the notion of viscosity solution.

Definition 1. (i) A function $u:[0, T] \times \mathbf{R}^{n} \rightarrow \mathbf{R}$ is a (viscosity) subsolution of (1) if $u$ is upper semicontinuous, $u(0, x) \leq u_{0}(x) \forall x \in \mathbf{R}^{n}$, and $\forall \varphi \in \mathbf{C}^{2}$, if $(\widehat{t}, \widehat{x}) \in[0, T] \times \mathbf{R}^{n}$ is a maximum of $u-\varphi$, then

$$
\varphi_{t}(\widehat{t}, \widehat{x})+\underline{F}\left(D \varphi(\widehat{t}, \widehat{x}), D^{2} \varphi(\widehat{t}, \widehat{x})\right)-k(\widehat{x})|D \varphi(\widehat{t}, \widehat{x})| \leq 0,
$$

where $\underline{F}$ is the lower semicontinuous envelope of $-F$.

(ii) A function $u:[0, T] \times \mathbf{R}^{n} \rightarrow \mathbf{R}$ is a (viscosity) supersolution of (1) if $u$ is lower semicontinuous, $u(0, x) \geq u_{0}(x) \forall x \in \mathbf{R}^{n}$, and $\forall \varphi \in \mathbf{C}^{2}$, if $(\widehat{t}, \widehat{x}) \in[0, T] \times \mathbf{R}^{n}$ is a minimum of $u-\varphi$, then

$$
\varphi_{t}(\widehat{t}, \widehat{x})+\bar{F}\left(D \varphi(\widehat{t}, \widehat{x}), D^{2} \varphi(\widehat{t}, \widehat{x})\right)-k(\widehat{x})|D \varphi(\widehat{t}, \widehat{x})| \geq 0,
$$

where $\bar{F}$ is the upper semicontinuous envelope of $-F$.

(iii) A function $u:[0, T] \times \mathbf{R}^{n} \rightarrow \mathbf{R}$ is a (viscosity) solution of (1) if $u$ is both a (viscosity) subsolution and a (viscosity) supersolution of (1). 
We recall that there are some other equivalent definitions expressed in terms of the socalled parabolic super 2-jets (see, e.g., [17] and [20]) but they will not be used in this paper.

The following existence and uniqueness of solutions result is a special case of Theorems 4.1 and 4.9 of [21]. We recall some previous notation: given a constant $\beta \in \mathbf{R}$ and a metric space $K, C_{\beta}(K)$ denotes the space of continuous functions $v$ such that $v()-.\beta$ is compactly supported in $K$.

Proposition 2. Let $k(x)$ be a bounded Hölder continuous function and let $G$ be given by (11). Assume that $u_{0} \in C_{\beta}\left(\mathbf{R}^{n}\right)$ for some constant $\beta \in \mathbf{R}$. Then problem (1) has a unique viscosity solution $u \in C_{\beta}\left([0, T] \times \mathbf{R}^{n}\right)$. Moreover the comparison principle holds under the above conditions, i.e., if $\underline{u}(t, x)$ and $\bar{u}(t, x)$ are, respectively, a subsolution and a supersolution of (1) satisfying that $\underline{u}(0, x) \leq \bar{u}(0, x) \forall x \in \mathbf{R}^{n}$, then

$$
\underline{u}(t, x) \leq \bar{u}(t, x) \quad \forall(t, x) \in[0, T] \times \mathbf{R}^{n} .
$$

Proof. It reduces to check the assumptions made in Theorems 4.1 and of [21]. Keeping the same notation as in Theorem 4.1 of [21] applied to the operator $G(t, x, u, p, X)$ given by (11), it is obvious that

(i) $G(t, x, u, p, X)$ is a continuous function over $J_{0}:=[0, T] \times \mathbf{R}^{n} \times \mathbf{R} \times\left(\mathbf{R}^{n} \backslash\{0\}\right) \times \mathbf{S}^{n}$;

(ii) $G(t, x, u, p, X)$ is degenerate elliptic (thanks to (2));

(iii) $-\infty<\underline{F}(0, O)=\bar{F}(0, O)<+\infty$, where $\underline{F}$ is the lower semicontinuous envelope of $-F$ and $\bar{F}$ is the upper semicontinuous envelope of $-F$;

(iv) for every $R>0 c_{R}:=\sup \left\{|G(t, x, u, p, X)|:|p|,|X| \leq R,(t, x, u, p, X) \in J_{0}\right\}<\infty$ (since $k$ is bounded);

(v) $G(t, x, u, p, X)$ is independent on $u$;

(vi) for every $R>\rho$ there is a modulus $\sigma$ (i.e., $\sigma:[0, \infty) \rightarrow[0, \infty), \sigma(0)=0$, and $\alpha$ is nondecreasing), $\sigma=\sigma_{R_{\rho}}$, such that

$$
|G(t, x, u, p, X)-G(t, x, u, q, Y)| \leq \sigma_{R_{\rho}}(|p-q|+|X-Y|)
$$

$\forall(t, x, u) \in[0, T] \times \mathbf{R}^{n} \times \mathbf{R}, \rho \leq|p|,|q| \leq R,|X|,|Y| \leq R$ (again due to the boundedness of $k)$;

(vii) there are $\rho_{0}>0$ and a modulus $\sigma_{1}$ such that

$$
\begin{gathered}
\bar{F}(p, X)-\bar{F}(0, O)-k(x)|p| \leq \sigma_{1}(|p|+|X|), \\
\underline{F}(p, X)-\underline{F}(0, O)-k(x)|p| \geq-\sigma_{1}(|p|+|X|),
\end{gathered}
$$

provided $x \in \mathbf{R}^{n}$ and $|p|,|X| \leq \rho_{0}$ (once again by the boundedness of $k$ ); and

(viii) there is a modulus $\sigma_{2}$ such that

$$
|G(t, x, u, p, X)-G(t, y, u, q, Y)| \leq \sigma_{2}(|x-y|)(|p|+1)
$$

for $(t, x, u, p, X),(t, y, u, p, X) \in J_{0}\left(\right.$ take $\sigma_{2}(s)=M s^{\alpha}$ with $\alpha \in(0,1)$ the Hölder exponent of $k(x))$.

The assumptions of Theorem 4.9 of [21] require additionally the condition $G$ geometric, but this holds obviously since

$$
G(x, \lambda p, \lambda X+\sigma p \otimes p)=\lambda G(x, p, X) \quad \forall \lambda>0, \sigma \in \mathbf{R}, \forall p \in \mathbf{R}^{n} \text { and } \forall x \in \mathbf{R}^{n} .
$$


Remark 1. As a matter of fact, since the conclusion in the above result shows that $u \in$ $C_{\beta}\left([0, T] \times \mathbf{R}^{n}\right)$, we could consider also data $k(x)$ which are Hölder continuous but unbounded in $\mathbf{R}^{n}$. Indeed, in that case we can truncate function $k(x)$ outside a ball $B_{R}(0)$, with radius $R>0$ large enough, and apply Theorem 1 to this truncation $k_{R}(x)$ which now is bounded and Hölder continuous in $\mathbf{R}^{n}$. Obviously, the speed of propagation would increase with the "size" of the (compact) support of the initial data, but in any case the speed would be finite for any given $u_{0} \in C_{\beta}\left(\mathbf{R}^{n}\right)$.

An important property (direct consequence of the results of [18] and [16]), unique to the geometric flows, is that the composition of any solution with a nondecreasing function remains a solution of the equation. So we have the following useful corollary.

Corollary 3. If $u(t, x)$, is a bounded uniformly continuous viscosity solution of (1), then for any nondecreasing function $\phi: \mathbf{R} \rightarrow \mathbf{R}$ the function $v(t, x)=\phi(u(t, x))$ is a viscosity solution of (1).

Remark 2. In [25], [23], [24] the authors study the problem (1) with obstacles for a forcing term $k(x)$ assumed to be bounded and Lipschitz continuous. We conjecture that it seems possible to extend the existence and uniqueness of solutions also to this context and under the mere assumption of $k(x)$ being bounded Hölder continuous. We also mention that weaker assumptions on $k(x)$ (as $k \in L^{\infty}\left(\mathbf{R}^{n}\right)$ ) were assumed in [15] for the study of (1)-(4) in terms of the curve evolution context.

Remark 3. The assumption $u_{0}()-.\beta$ is compactly supported in $\mathbf{R}^{n}$ is not restrictive at all when we consider bounded hypersurface evolution. Indeed, if $\Gamma_{0}$ is a bounded hypersurface, using the signed distance function we can embed $\Gamma_{0}$ in a function $u_{0} \in C_{\beta}\left(\mathbf{R}^{n}\right)$. Moreover, as we shall see in the next section, the hypersurface evolution is independent of the particular choice of function $u_{0}$ where $\Gamma_{0}$ is embedded and therefore the size of the support of $u_{0}()-.\beta$ is not relevant in terms of the hypersurface evolution.

3. Hypersurface evolution using the parabolic perturbed mean curvature equation. We will consider an initial hypersurface $\Gamma_{0}$ of $\mathbf{R}^{n}$ as the boundary of a bounded open set $U_{0}$. We choose any $u_{0}: \mathbf{R}^{n} \rightarrow \mathbf{R}$ such that $u_{0} \in C_{\beta}\left(\mathbf{R}^{n}\right)$ for some constant $\beta \in \mathbf{R}$ satisfying

$$
u_{0}(x)=\left\{\begin{array}{ccc}
u_{0}(x)<0 & \text { if } & x \in U_{0}, \\
u_{0}(x)>0 & \text { if } & x \in\left(\mathbf{R}^{n}-\overline{U_{0}}\right), \\
u(x)=0 & \text { if } & x \in \Gamma_{0}=\partial U_{0} .
\end{array}\right.
$$

It is not difficult to see [20] that, in fact, we can choose as $u_{0}$ any bounded uniformly continuous function if the datum $k(x)$ is Lipschitz continuous. Then, we define $\left\{U_{t}\right\}_{t \geq 0}$ and $\left\{\Gamma_{t}\right\}_{t>0}$ as

$$
U_{t}=\left\{x \in \mathbf{R}^{n}: u(t, x)<0\right\},
$$

$$
\Gamma_{t}=\partial U_{t},
$$

where $u(t, x)$ is the (unique) viscosity solution of (1) for the initial datum $u_{0}(x)$. One important remark is that, in contrast to the case where no forcing term is included $(k \equiv 0)$, the sign 
of $u_{0}(x)$ in $U_{0}$ matters in the evolution of $U_{0}$. Indeed if $u(t, x)$ is a solution of (1)-(4), then $v(t, x)=-u(t, x)$ is the solution of (1)-(4) but changing $k(x)$ by $-k(x)$ and therefore $U_{t}, \Gamma_{t}$ will be different using $u_{0}(x)$ or $-u_{0}(x)$ as initial surface to embed $\Gamma_{0}$.

Let us prove first that $U_{t}$ and $\Gamma_{t}$ are well defined in the sense that they are independent of the choice of $u_{0}$.

Theorem 4. Let $U_{0}$ be an open bounded set, $\Gamma_{0}=\partial U_{0}, F$ satisfying (2)-(3), and $u_{0} \in$ $C_{\beta}\left(\mathbf{R}^{n}\right), \hat{u}_{0} \in C_{\widehat{\beta}}\left(\mathbf{R}^{n}\right)$, for some constants $\beta, \widehat{\beta} \in \mathbf{R}$, satisfying (14). Then, for any $t>0$

$$
\left\{x \in \mathbf{R}^{n}: u(t, x)<0\right\}=\left\{x \in \mathbf{R}^{n}: \hat{u}(t, x)<0\right\},
$$

where $u(t, x), \hat{u}(t, x)$ are the viscosity solutions of (1) associated to the initial data $u_{0}(x), \hat{u}_{0}(x)$.

Proof. This proof is based on the techniques presented in [18], [23]. First, we are going to show that if $u_{0}(x), \hat{u}_{0}(x)$ satisfy (14), then we can build a nondecreasing function $\phi: \mathbf{R} \rightarrow \mathbf{R}$ satisfying that $\{\phi \equiv 0\}=\{0\}$ and $\hat{u}_{0}(x) \leq \phi\left(u_{0}(x)\right)$. We define $E_{0}=\mathbf{R}^{n}$ and $E_{m}=\left\{x \in \mathbf{R}^{n}\right.$ : $\left.u_{0}(x) \leq \frac{1}{m}\right\}$, for $m \in \mathbf{Z}-\{0\}$, then we have for $m>0$

$$
E_{-1} \subseteq \cdots \subseteq E_{-m} \subseteq \cdots \subset \overline{U_{0}} \subset \cdots \subseteq E_{m} \subseteq \cdots \subseteq E_{0} ;
$$

next we define $a_{0}=\sup _{x \in \mathbf{R}^{n}} \hat{u}_{0}$ and

$$
a_{m}=\left\{\begin{array}{ccc}
\sup _{x \in E_{m-1}} \hat{u}_{0} & \text { if } & E_{m-1} \neq \varnothing, \\
\inf _{x \in R^{n}} \hat{u}_{0} & \text { if } & E_{m-1}=\varnothing
\end{array}\right.
$$

for $m \in \mathbf{Z}-\{0\}$. Using (14)-(17) we obtain for $m>0$

$$
a_{-1} \leq \cdots \leq a_{-m} \leq \cdots<0<\cdots \leq a_{m} \leq \cdots \leq a_{1} .
$$

Finally, we define $\phi(s)$ to be a continuous nondecreasing function satisfying

$$
\begin{gathered}
\phi(0)=0, \\
\phi\left(\frac{1}{m}\right)=a_{m} \quad \forall m \in \mathbf{Z}-\{0\}, \\
\phi \text { linear on }\left[\frac{1}{m+1}, \frac{1}{m}\right], \quad m=1,2, \ldots, \\
\phi \text { linear on }\left[\frac{1}{m}, \frac{1}{m-1}\right], \quad m=-1,-2, \ldots, \\
\phi \text { constant outside }[-1,1],
\end{gathered}
$$

and by construction, we can easily show that $\hat{u}_{0}(x) \leq \phi\left(u_{0}(x)\right)$ and by the comparison principle shown in Proposition 2 we obtain

$$
\hat{u}(t, x) \leq \phi(u(t, x))
$$

and then

$$
\left\{x \in \mathbf{R}^{n}: \hat{u}(t, x)<0\right\} \subseteq\left\{x \in \mathbf{R}^{n}: \phi(u(t, x))<0\right\}=\left\{x \in \mathbf{R}^{n}: u(t, x)<0\right\} .
$$

The opposite inclusion is proven in a similar way switching $u(t, x)$ and $\hat{u}(t, x)$.

Let $U_{0}$ be a bounded open set, $\Gamma_{0}=\partial U_{0}$. A typical choice of function $u_{0}(x)$ satisfying (14) is the following truncated signed distance function:

$$
d_{U_{0}, \beta}(x)=\left\{\begin{array}{clc}
-\operatorname{dist}\left(x, \Gamma_{0}\right) & \text { if } & x \in \overline{U_{0}} \\
\operatorname{dist}\left(x, \Gamma_{0}\right) & \text { if } & x \in\left(\mathbf{R}^{n}-\overline{U_{0}}\right) \wedge \operatorname{dist}\left(x, \Gamma_{0}\right) \leq \beta \\
\beta & & \text { otherwise }
\end{array}\right.
$$


for some $\beta>0$. We point out that if $U_{0}$ is bounded, then $d_{U_{0}, \beta} \in C_{\beta}\left(\mathbf{R}^{n}\right)$. Moreover, following the results of the above theorem, the evolution of $\Gamma_{0}$ is independent of the choice of $\beta>0$ used to define the truncated signed distance. Next we show that the comparison principle holds for $U_{t}$.

Corollary 5. Let $U_{0}, \hat{U}_{0}$ be bounded open sets satisfying $U_{0} \subseteq \hat{U}_{0}$; then for $t \geq 0 U_{t} \subseteq \hat{U}_{t}$.

Proof. Using Theorem 4, without loss of generality, we can assume that $u_{0}(x)$ and $\hat{u}_{0}(x)$ are given by the distance function (18). If $U_{0} \subseteq \hat{U}_{0}$, then

$$
\hat{u}_{0}(x) \leq u_{0}(x),
$$

and by the comparison of the viscosity solutions given in Proposition 2, we obtain that

$$
U_{t}=\left\{x \in \mathbf{R}^{n}: u(t, x)<0\right\} \subseteq\left\{x \in \mathbf{R}^{n}: \hat{u}(t, x)<0\right\}=\hat{U}_{t},
$$

which completes the proof.

4. Radial solutions of the parabolic perturbed mean curvature equation. In the case $U_{0}$ is a ball, $B_{r_{0}}\left(x_{0}\right)$, of radius $r_{0}$, then the truncated signed distance function (18) becomes

$$
d_{B_{r_{0}}\left(x_{0}\right), \beta}(x)=\left\{\begin{array}{ccc}
\left|x-x_{0}\right|-r_{0} & \text { if } & \left|x-x_{0}\right|<r_{0}+\beta \\
\beta & \text { otherwise }
\end{array}\right.
$$

Let $u(t, r)$ be a radial solution of (1) with $F$ given by (4). In radial coordinates this equation becomes

$$
u_{t}(t, r)=\left(\frac{n-1}{r} \operatorname{sgn}\left(u_{r}(t, r)\right)+k(r)\right)\left|u_{r}(t, r)\right|,
$$

where $\operatorname{sgn}($.$) is the sign function. In this case U_{t}$ is given by the ball $B_{r(t)}\left(x_{0}\right)$, where $r(t)$ satisfies

$$
u(t, r(t))=0,
$$

and by computing the derivatives of this expression we obtain

$$
u_{t}(t, r(t))+\frac{d r}{d t}(t) u_{r}(t, r(t))=0 .
$$

Using (20)-(22) and assuming that $u_{r}(t, r(t))>0$ (the case $u_{r}(t, r(t))<0$ is similar) we obtain that $r(t)$ satisfies the equation

$$
\frac{d r}{d t}(t)=-\frac{n-1}{r(t)}-k(r(t)) .
$$

Notice, once again, that the mere assumption of $k$ Hölder continuous is enough for the correct treatment of this ODE. Therefore by a straightforward integration we obtain that if

$$
-\frac{n-1}{r(t)}-k(r(t)) \neq 0 \text { for } r(t) \in(a, b),
$$


then there exists a strictly monotone function $\psi:(a, b) \rightarrow R$ such that

$$
\psi^{\prime}(r)=\frac{1}{-\frac{n-1}{r}-k(r)} \quad \forall r \in(a, b),
$$

and for any $r_{0} \in(a, b)$,

$$
r(t)=\eta\left(t+\psi\left(r_{0}\right)\right) \quad \text { for } t \in\left[0, t^{*}\right],
$$

where $\eta \equiv \psi^{-1}$ and $t^{*}=\sup \left\{t: \eta\left(t+\psi\left(r_{0}\right)\right) \in(a, b)\right\}$.

Remark 4. We point out that if we know the evolution of the radius of the ball, $r(t)$, for any $r_{0}>0$, then we can construct an explicit formula for the solution of (1) with $F$ given by (4). Indeed, for $-r_{0}<c<r_{0}+1$ we define $U_{0}^{c}=\left\{x: d_{B_{r_{0}}\left(x_{0}\right), \beta}(x)<c\right\}=B_{r_{0}+c}\left(x_{0}\right)$ and $U_{t}^{c}=B_{r^{c}(t)}\left(x_{0}\right)$, where $r^{c}(t)$ is the solution of (23) for the radius $r_{0}+c$. Then we can recover the solution of (1)-(4) using the expression

$$
u(t, x)=\inf \left\{c: x \in B_{r^{c}(t)}\left(x_{0}\right)\right\} .
$$

Let us study the shape of $r(t)$, the solution of (23), for some particular choices of the forcing term $k(r)$. The proofs of the following lemmas are straighforward using basic integration techniques.

Lemma 6 (case $k(x)$ constant). Let $k \equiv k_{0} \in \mathbf{R}-\{0\}$ and let $r(t)$ be the solution of (23). (i) If $k_{0}>0$, then

$$
r(t)=\left\{\begin{array}{llr}
\eta\left(t+\psi\left(r_{0}\right)\right) & \text { if } & t \in\left[0, \psi(0)-\psi\left(r_{0}\right)\right) \\
0 & \text { if } & t \geq \psi(0)-\psi\left(r_{0}\right)
\end{array}\right.
$$

where $\eta \equiv \psi^{-1}$ with $\psi:[0, \infty) \rightarrow(-\infty, \psi(0)]$ the decreasing function defined by

$$
\psi(r)=\frac{n-1}{k_{0}^{2}} \ln \frac{(n-1)+r k_{0}}{\left|k_{0}\right|}-\frac{r}{k_{0}} .
$$

(ii) If $k_{0}<0$, then

$$
\begin{array}{llrl}
r(t)=\eta_{1}\left(t+\psi_{1}\left(r_{0}\right)\right) & \text { if } & r_{0}<\frac{1-n}{k_{0}} \text { and } t \in\left[0, \psi_{1}(0)-\psi_{1}\left(r_{0}\right)\right), \\
r(t)=0 & \text { if } & r_{0}<\frac{1-n}{k_{0}} \text { and } t \geq \psi_{1}(0)-\psi_{1}\left(r_{0}\right), \\
r(t) \equiv r_{0} & \text { if } & r_{0}=\frac{1-n}{k_{0}} \text { and } t \geq 0, \\
r(t)=\eta_{2}\left(t+\psi_{2}\left(r_{0}\right)\right) & \text { if } & r_{0}>\frac{1-n}{k_{0}} \text { and } t \geq 0,
\end{array}
$$

where $\psi_{1}:\left[0, \frac{1-n}{k_{0}}\right) \rightarrow\left(-\infty, \psi_{1}(0)\right]$ is a decreasing function, $\psi_{2}:\left(\frac{1-n}{k_{0}}, \infty\right) \rightarrow(-\infty, \infty)$ is an increasing function, $\eta_{1}=\psi_{1}^{-1}, \eta_{2}=\psi_{2}^{-1}$, and

$$
\begin{array}{lll}
\psi_{1}(r)=\frac{n-1}{k_{0}^{2}} \ln \frac{1-n-r k_{0}}{k_{0}}-\frac{r}{k_{0}} \quad \text { if } \quad r_{0}<\frac{1-n}{k_{0}}, \\
\psi_{2}(r)=\frac{n-1}{k_{0}^{2}} \ln \frac{n-1+r k_{0}}{k_{0}}-\frac{r}{k_{0}} \quad \text { if } \quad r_{0}>\frac{1-n}{k_{0}} .
\end{array}
$$

In Figure 1, we show the profile of $\eta_{1}(s)$ and $\eta_{2}(s)$ for $k(r) \equiv-1$ and $n=2$. 


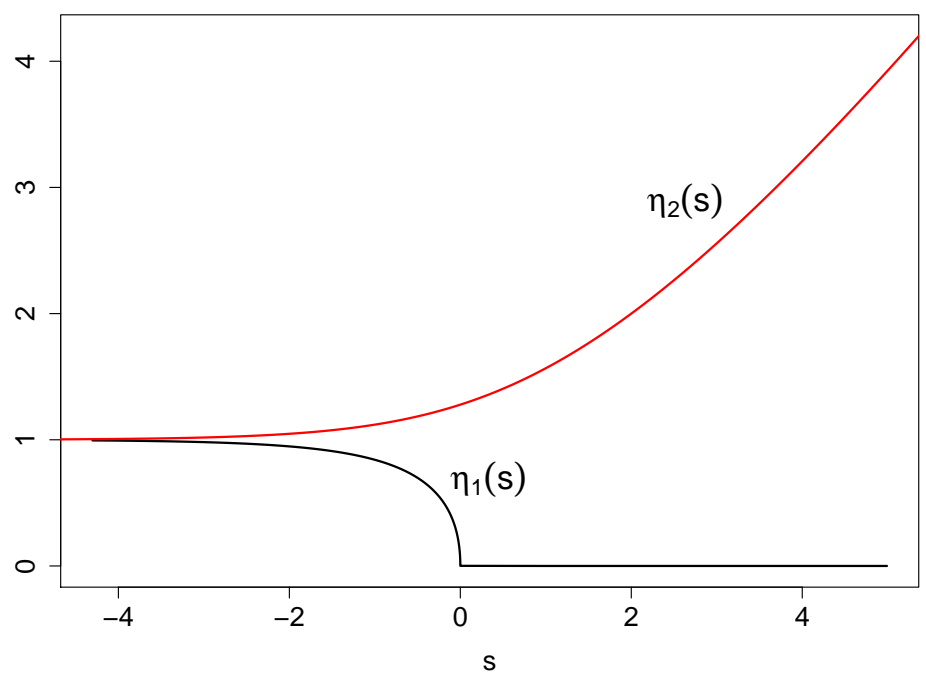

Figure 1. Plot of $\eta_{1}(s)$ and $\eta_{2}(s)$ for $k(r) \equiv-1$ and $n=2$.

Remark 5. We point out that according to Lemma 6 , if $k_{0}$ is positive, or $k_{0}$ is negative and $r_{0}<\frac{1-n}{k_{0}}$, then $r(t)$ is a decreasing function which vanishes in finite time. This is the typical behavior of radial solutions in the case of the equation without forcing term $(k \equiv 0)$ : see, e.g., [2]. That is, the ball $B_{r(t)}\left(x_{0}\right)$ tends to shrink across the time. However, in the case $k_{0}$ is negative and $r_{0}>\frac{1-n}{k_{0}}$, then $r(t)$ is an increasing function such that $\lim _{t \rightarrow \infty} r(t)=\infty$. That is, the ball $B_{r(t)}\left(x_{0}\right)$ tends to expand across the time.

Lemma 7 (case $k(r)$ linear). Assume $k(r) \equiv m r+c$ for $r \in\left[0, r^{*}\right]$ with $m>0, c<0$, and $r^{*}>\max \left\{r_{0},-m / c\right\}$. (i) If $-4 m(n-1)+c^{2}>0$, then the function $v(r)=-\frac{n-1}{r}-m r-c$ has the roots $R_{1}>R_{0}>0$ given by

$$
\begin{aligned}
& R_{0}=R_{0}(n, m, c)=\frac{1}{2}\left(\frac{-c}{m}-\sqrt{\frac{4(n-1)}{-m}+\left(\frac{c}{m}\right)^{2}}\right) \\
& R_{1}=R_{1}(n, m, c)=\frac{1}{2}\left(\frac{-c}{m}+\sqrt{\frac{4(n-1)}{-m}+\left(\frac{c}{m}\right)^{2}}\right) .
\end{aligned}
$$

In consequence, the solution $r(t)$ of $(23)$ is given by $r(t)=\eta\left(t+\psi\left(r_{0}\right)\right)$, where $\eta \equiv \psi^{-1}$ with

$$
\psi(r)=\ln \left(\left|r-R_{0}\right|^{\frac{1}{2 m} \frac{-c-\sqrt{-4 m(n-1)+c^{2}}}{\sqrt{-4 m(n-1)+c^{2}}}}\left|r-R_{1}\right|^{-\frac{1}{2 m} \frac{-c+\sqrt{-4 m(n-1)+c^{2}}}{\sqrt{-4 m(n-1)+c^{2}}}}\right) .
$$

(ii) Assume $-4 m(n-1)+c^{2}=0$. Then $r(t)=\eta\left(t+\psi\left(r_{0}\right)\right)$, where $\eta \equiv \psi^{-1}$ with

$$
\psi(r)=\frac{(-c-2 m r) \ln \left(\left|\frac{c+2 m r}{2 m}\right|\right)-c}{m(c+2 m r)} .
$$




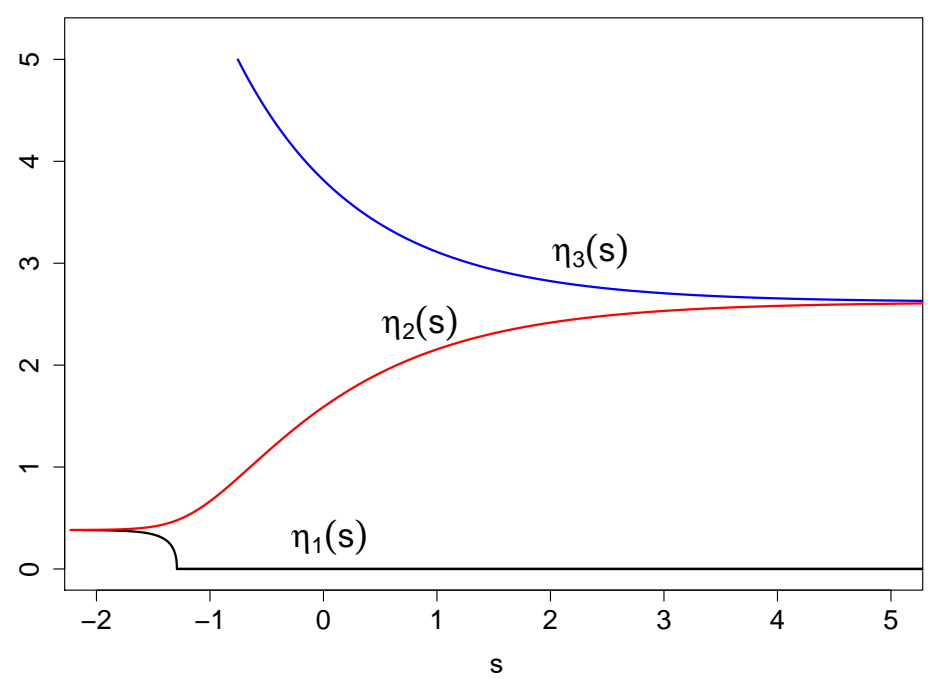

Figure 2. (Case (i) of Lemma 7). Plot of $\eta_{1}(s), \eta_{2}(s)$, and $\eta_{3}(s)$ for $k(r)=r-3$ and $n=2$.

(iii) Assume $-4 m(n-1)+c^{2}<0$. Then $r(t)=\eta\left(t+\psi\left(r_{0}\right)\right)$, where $\eta \equiv \psi^{-1}$ with

$$
\begin{gathered}
\psi(r)=-\frac{1}{2(m)^{\frac{3}{2}}} \frac{b}{\sqrt{\frac{-4 m(n-1)+c^{2}}{-4 m}}} \arctan \left(\sqrt{\frac{-4 m}{-4 m(n-1)+c^{2}}} \sqrt{m} r+\sqrt{\frac{-4 m}{-4 m(n-1)+c^{2}}} \frac{c}{2 \sqrt{m}}\right) \\
-\frac{1}{2 m} \ln \left|n-1+m r^{2}+c r\right| .
\end{gathered}
$$

Remark. We notice that as in Lemma 6, functions $\psi(r)$ and $\eta(r)$ should be distinguished on intervals where $\psi(r)$ changes its monotonicity. Therefore, in practice, we have to deal with $\psi_{i}(r)$ and $\eta_{i}(r)=\psi_{i}^{-1}(r)$ in different intervals. In Figures 2, 3, and 4, we show the profile of functions $\eta_{i}(r)$ for the different cases of the previous lemma.

Remark. We point out that the solution $r(t)$ of (23) is given by $r(t)=\eta_{i}\left(t+\psi_{i}\left(r_{0}\right)\right)$ and satisfies the following qualitative properties:

In case (i) of Lemma 7,

$$
\left\{\begin{array}{clc}
\lim _{t \rightarrow \infty} r(t)=R_{1} & \text { if } & r_{0} \in\left(R_{1}, r^{*}\right), \\
r(t) \equiv R_{1} & \text { if } & r_{0}=R_{1}, \\
\lim _{t \rightarrow \infty} r(t)=R_{1} & \text { if } & r_{0} \in\left(R_{0}, R_{1}\right), \\
r(t) \equiv R_{0} & \text { if } & r_{0}=R_{0}, \\
\lim _{t \rightarrow t_{\infty} r(t)=0} & \text { if } & r_{0}<R_{0} \text { with } t_{\infty}=\psi(0)-\psi\left(r_{0}\right), \\
r(t)=0 & \text { if } & r_{0}<R_{0} \text { and } t \geq t_{\infty} .
\end{array}\right.
$$

In case (ii) of Lemma 7 ,

$$
\left\{\begin{array}{clc}
\lim _{t \rightarrow \infty} r(t)=R_{1} & \text { if } & r_{0} \in\left(R_{0}, r^{*}\right) \\
r(t) \equiv R_{0} & \text { if } & r_{0}=R_{0} \\
\lim _{t \rightarrow t_{\infty}} r(t)=0 & \text { if } & r_{0}<R_{0} \text { with } t_{\infty}=\psi(0)-\psi\left(r_{0}\right), \\
r(t)=0 & \text { if } & r_{0}<R_{0} \text { and } t \geq t_{\infty}
\end{array}\right.
$$




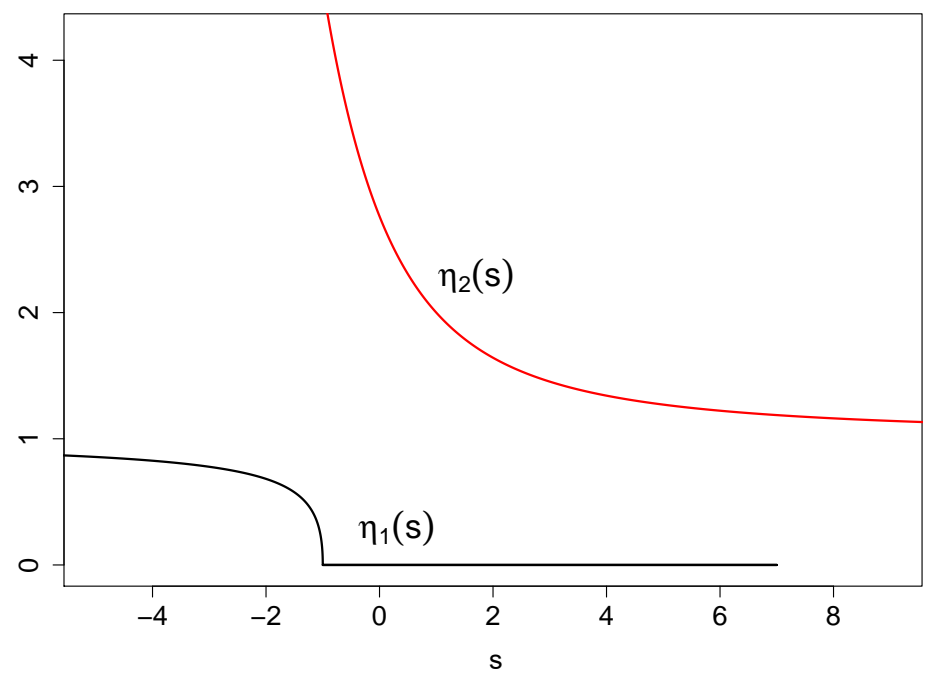

Figure 3. (Case (ii) of Lemma 7.) Plot of $\eta_{1}(s)$ and $\eta_{2}(s)$ for $k(r)=r-2$ and $n=2$.

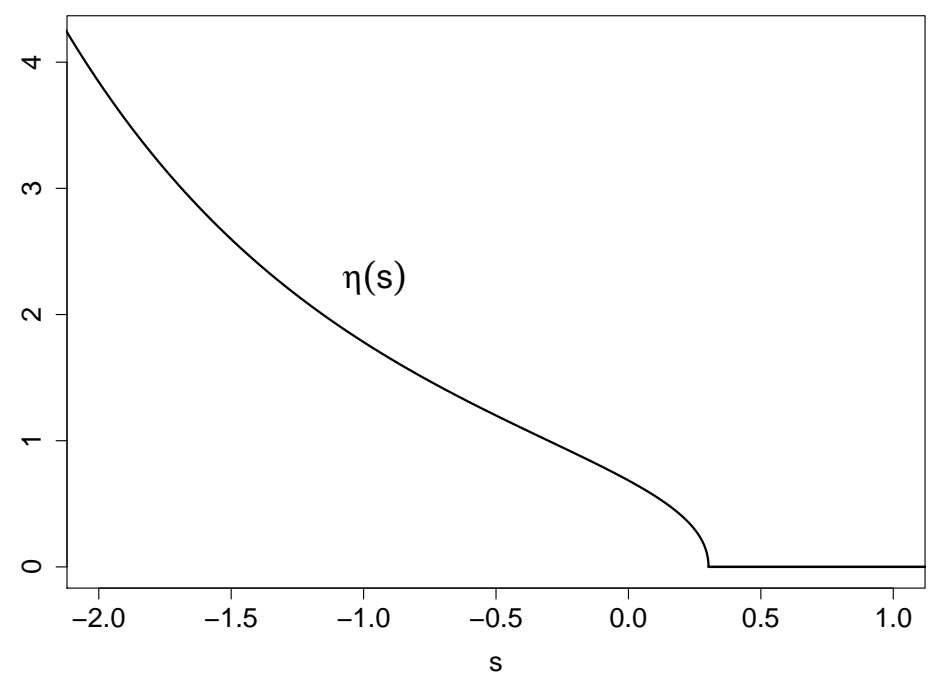

Figure 4. (Case (iii) of Lemma 7.) Plot of $\eta(s)$ for $k(r)=r-1$ and $n=2$.

In case (iii) of Lemma 7,

$$
\left\{\begin{array}{clc}
\lim _{t \rightarrow t_{\infty}} r(t)=0 & \text { if } & r_{0} \in\left(0, r^{*}\right) \text { with } t_{\infty}=\psi(0)-\psi\left(r_{0}\right) \\
r(t)=0 & \text { if } & t \geq t_{\infty}
\end{array}\right.
$$

As mentioned before, in case (ii) of Lemma 7 we observe that if $r_{0}<R_{0}$, then $r(t)$ converges in finite time to 0 and $\lim _{t \rightarrow \infty} r(t)=R_{0}$ when $r_{0}>R_{0}$. One interesting point is to know how to choose the forcing term $k(r)$ in order to have $r(t)$ converging to $R_{0}$ in finite time (assumed $r_{0}>R_{0}$ ). In the next lemma we study this issue. 
Lemma 8 (stabilization in finite time of radial solutions). Let $r^{*}, R_{0}$ be such that $r^{*}>R_{0}>0$ and $k(r)$ satisfying

$$
\begin{gathered}
-\frac{n-1}{r}-k(r)<0 \quad \text { for } r \in\left(R_{0}, r^{*}\right), \\
-\frac{n-1}{R_{0}}-k\left(R_{0}\right)=0, \\
0<-\int_{R_{0}}^{r^{*}} \frac{d r}{-\frac{n-1}{r}-k(r)}<\infty .
\end{gathered}
$$

Then, for any $r_{0} \in\left(R_{0}, r^{*}\right)$, the solution of (23) is given by

$$
r(t)=\left\{\begin{array}{cl}
\eta\left(t+\psi\left(r_{0}\right)\right) & \text { if } t \in\left[0, \psi\left(R_{0}\right)-\psi\left(r_{0}\right)\right] \\
R_{0} & \text { if } \quad t>\psi\left(R_{0}\right)-\psi\left(r_{0}\right)
\end{array}\right.
$$

where $\eta \equiv \psi^{-1}$ with $\psi(r)$ given by decreasing function

$$
\psi(r)=\int_{R_{0}}^{r} \frac{d s}{-\frac{(n-1)}{s}-k(s)} .
$$

In particular, the above conditions are fulfilled for the following Hölder continuous function:

$$
k(r)=-\frac{n-1}{R_{0}}+C\left(r-R_{0}\right)^{\gamma} \quad \forall r \in\left[R_{0}, r^{*}\right]
$$

with $\gamma \in(0,1)$ and $C>0$ big enough. In fact, for any $R_{0}>0$ and $\gamma \in(0,1)$ there exists $C^{*}>0$ such that, if $C \geq C^{*}$, function

$$
k_{R_{0}, \gamma, C}(r)=\left\{\begin{array}{ccc}
\frac{n-1}{R_{0}^{2}}\left(r-R_{0}\right)-\frac{n-1}{R_{0}} & \text { if } & r<R_{0}, \\
-\frac{n-1}{R_{0}}+C\left(r-R_{0}\right)^{\gamma} & \text { if } & r \in\left[R_{0}, r^{*}\right]
\end{array}\right.
$$

satisfies conditions (29)-(30)-(31) for $r \in\left[R_{0}, r^{*}\right], k(r)=m r+c$, with $c^{2}=4 m(n-1)$ and $R_{0}=\frac{-c}{2 m}$, for $r<R_{0}$. Therefore the corresponding solution of (23) is given by $r(t)=$ $\eta\left(t+\psi\left(r_{0}\right)\right)$ as before and verifies

$$
\left\{\begin{array}{clc}
\lim _{t \rightarrow t_{\infty}^{+}} r(t)=R_{0} & \text { if } & r_{0} \in\left(R_{0}, r^{*}\right) \text { with } t_{\infty}^{+}=\psi\left(R_{0}\right)-\psi\left(r_{0}\right), \\
r(t) \equiv R_{0} & \text { if } & r_{0} \in\left[R_{0}, r^{*}\right) \text { and } t \geq t_{\infty}^{+}, \\
\lim _{t \rightarrow t_{\infty}^{-}} r(t)=0 & \text { if } & r_{0}<R_{0} \text { with } t_{\infty}^{-}=\psi(0)-\psi\left(r_{0}\right), \\
r(t)=0 & \text { if } & r_{0}<R_{0} \text { and } t \geq t_{\infty}^{-} .
\end{array}\right.
$$

Remark. We point out that the function $k(r)$ given by (32) is Hölder but not Lipschitz continuous since $\lim _{r \downarrow R_{0}} k^{\prime}(r)=\infty$. In fact, it is easy to show (as in [2]) that if, for $R_{0}>0$, conditions (30)-(31) are satisfied, then $k(r)$ cannot be Lipschitz in $\left[R_{0}, r^{*}\right)$. 


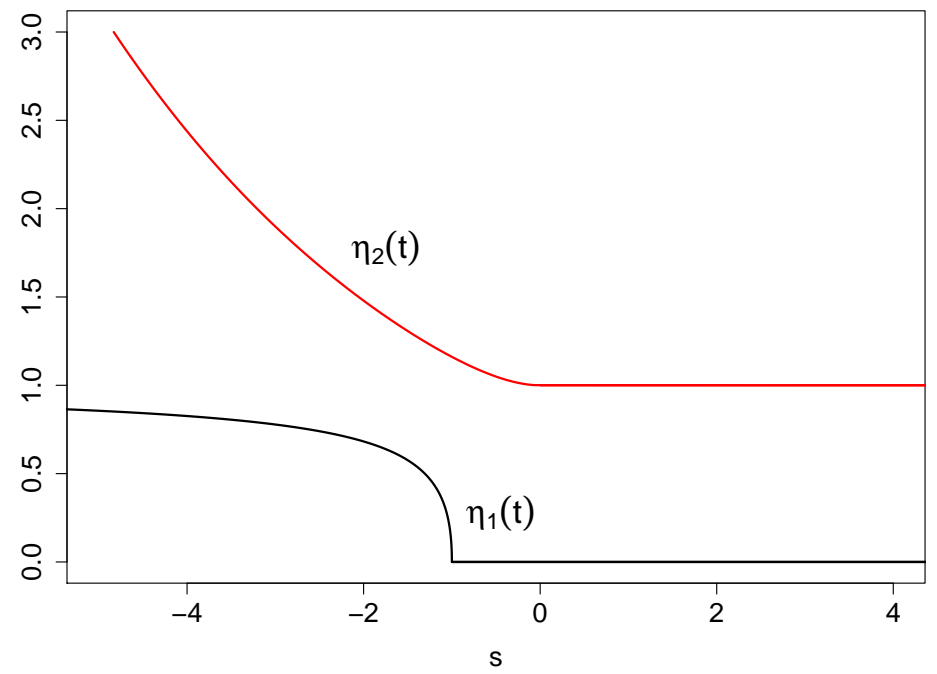

Figure 5. Plot of $\eta_{1}(s)$ and $\eta_{2}(s)$ for $k(r)$ given by (32) with $R_{0}=1, m=1, c=-2, \gamma=1 / 2, C=1$, and $n=2$.

\section{Some qualitative properties of the evolution of $U_{t}, \Gamma_{t}$.}

Theorem 9. Let $\left\{U_{t}\right\}_{t \geq 0}$ be the set evolution given by (15) under the action of the geometric flow (1)-(4) and $k_{0}=\min _{x \in \mathbf{R}^{n}} k$. Then if for some $x_{0} \in \mathbf{R}^{n}$ and $r_{0}>0, U_{0} \subseteq B_{r_{0}}\left(x_{0}\right)$, then $U_{t} \subseteq B_{r(t)}\left(x_{0}\right) \forall t \geq 0$, where $r(t)$ is given by (26) or (27) according to the sign of $k_{0}$.

Proof. Let $v(t, x)$ be the corresponding solution of the equation

$$
\frac{\partial v}{\partial t}=\operatorname{div}\left(\frac{\nabla v}{|\nabla v|}\right)|\nabla v|+k_{0}|\nabla v|
$$

with the same initial datum as indicated before. Then $v$ is a subsolution of the original problem and, by the comparison principle given in Theorem 1, we conclude that $U_{t} \subseteq B_{r(t)}\left(x_{0}\right)$.

Remark 6. From the above theorem we obtain that $U_{t}$ is bounded $\forall t \geq 0$ and that $B_{r(t)}\left(x_{0}\right)$ provides an upper estimate of the set evolution. In particular, according to the expressions (26)-(27), $U_{t}$ vanishes in finite time if $k_{0}$ is positive or if $k_{0}$ is negative and $r_{0}<\frac{1-n}{k_{0}}$. Therefore, in any case, if $U_{0}$ is small enough, $U_{t}$ vanishes in a finite time.

Theorem 10. Let $\left\{U_{t}^{1}\right\}_{t \geq 0},\left\{U_{t}^{2}\right\}_{t \geq 0}$ be two set evolutions given by (15) under the action of the geometric flow (1)-(4). If $\overline{U_{t}^{1}} \cap \overline{U_{t}^{2}}=\varnothing$ for $t \in[0, T]$, then the evolution level set $U_{t}$ for $U_{0}=U_{0}^{1} \cup U_{0}^{2}$ is given by $U_{t}=U_{t}^{1} \cup U_{t}^{2}$ for $t \in[0, T]$.

Proof. Let $u^{1}(t, x)$ and $u^{2}(t, x)$ be the solutions of (1)-(4) taking as initial data the distance function (18) for $U_{0}$ and $U_{1}$, respectively. By the definition of the distance function (18) and Theorem 9 we obtain that for $\epsilon<1, \overline{U_{t}^{1, \epsilon}}=\left\{x \in \mathbf{R}^{n}: u^{1}(t, x) \leq \epsilon\right\}$ and $\overline{U_{2}^{1, \epsilon}}=\left\{x \in \mathbf{R}^{n}\right.$ : $\left.u^{2}(t, x) \leq \epsilon\right\}$ are compact sets in $\mathbf{R}^{n}$. We are going to show that if $\epsilon$ is small enough, then $\overline{U_{t}^{1, \epsilon}} \cap \overline{U_{t}^{2, \epsilon}}=\varnothing$ for $t \in[0, T]$. Indeed, if we assume the opposite, that is, that for any $\epsilon<1$ there exists $\left(t_{\epsilon}, x_{\epsilon}\right) \in \overline{U_{t}^{1, \epsilon}} \cap \overline{U_{t}^{2, \epsilon}}$, then as $\left\{\left(t_{\epsilon}, x_{\epsilon}\right)\right\}_{\epsilon<1}$ is a bounded sequence, there exists a 
subsequence $\epsilon_{n} \downarrow 0$ such that $\left(t_{\epsilon_{n}}, x_{\epsilon_{n}}\right) \rightarrow\left(t^{*}, x^{*}\right)$ with $t^{*} \in[0, T]$. Then $u^{1}\left(t^{*}, x^{*}\right) \leq 0$ and $u^{2}\left(t^{*}, x^{*}\right) \leq 0$ and therefore $\left(t^{*}, x^{*}\right) \in \overline{U_{t}^{1}} \cap \overline{U_{t}^{2}}$, which is in contradiction with the assumption $\overline{U_{t}^{1}} \cap \overline{U_{t}^{2}}=\varnothing$. Let us take $\epsilon>0$ such that $\overline{U_{t}^{1, \epsilon}} \cap \overline{U_{t}^{2, \epsilon}}=\varnothing$. We define the nondecreasing function

$$
\phi_{\epsilon}(s)=\left\{\begin{array}{lll}
s & \text { if } \quad s<\epsilon \\
\epsilon & \text { if } \quad s \geq \epsilon
\end{array}\right.
$$

Then, for any $\epsilon>0$, functions $\phi_{\epsilon}\left(u^{1}(t, x)\right)$ and $\phi_{\epsilon}\left(u^{2}(t, x)\right)$ are solutions of (1)-(4). We point out that as $\overline{U_{t}^{1, \epsilon}}, \overline{U_{t}^{2, \epsilon}}$ are compact sets, then the distance between them is positive and then the function defined by

$$
u(t, x)=\left\{\begin{array}{ccc}
\phi_{\epsilon}\left(u^{1}(t, x)\right) & \text { if } & \phi_{\epsilon}\left(u^{1}(t, x)\right)<\epsilon \\
\phi_{\epsilon}\left(u^{2}(t, x)\right) & \text { if } & \phi_{\epsilon}\left(u^{2}(t, x)\right)<\epsilon \\
\epsilon & & \text { otherwise }
\end{array}\right.
$$

is well defined for $t \in[0, T]$, it is a solution of (1)-(4), and it satisfies that

$$
\left\{(t, x) \in[0, T] \times \mathbf{R}^{n}: u(t, x)<0\right\}=U_{t}^{1} \cup U_{t}^{2} .
$$

This concludes the proof of the theorem.

Remark 7. From the above theorem we obtain that, for small times, the evolution of the set $U_{t}$ is local in the sense that if $U_{0}=\cup_{k} U_{0}^{k}$, where $\left\{U_{0}^{k}\right\}$ are connected sets such that $\operatorname{distance}\left(U_{0}^{k}, U_{0}^{k^{\prime}}\right)>\delta>0$ for $k \neq k^{\prime}$, then $\overline{U_{t}^{k}} \cap \overline{U_{t}^{k^{\prime}}}=\varnothing$ for $t>0$ small enough. In consequence, each connected set $U_{0}^{k}$ evolves in an independent way during the interval $\left[0, t^{*}\right]$ with $t^{*}$ such that distance $\left(\overline{U_{t}^{k}}, U_{t}^{k^{\prime}}\right)>0 \forall t \in\left[0, t^{*}\right]$.

6. Numerical discretization of (1)-(4). The discretization scheme we propose is based on the general approach introduced in [26]. More sophisticated implicit numerical schemes for mean curvature motion have been proposed in [31]. We use an explicit finite difference method where the mean curvature term is discretized using central differencing and the forcing term is discretized using the upwind scheme proposed in [26] (sections 6.2 and 6.3). A more detailed study about this kind of upwind scheme for first order geometric flows has been presented in [9].

Let $t_{n}=n \cdot \Delta t$ and $\left\{x^{k}\right\}$ be the time and spatial discretization lattice. The ways the forcing term and the mean curvature term are discretized is different. For the forcing term we use the following upwind scheme:

$$
k_{m, k}|\nabla u|\left(t_{m}, x^{k}\right) \approx \begin{cases}k_{m, k} \sqrt{\sum_{i=1}^{n}\left(\max \left\{\max \left\{u_{x_{i}}^{+}, 0\right\}, \min \left\{u_{x_{i}}^{-}, 0\right\}\right\}\right)^{2}} & \text { if } \quad k_{m, k} \geq 0, \\ k_{m, k} \sqrt{\sum_{i=1}^{n}\left(\max \left\{\min \left\{u_{x_{i}}^{+}, 0\right\}, \max \left\{u_{x_{i}}^{-}, 0\right\}\right\}\right)^{2}} \quad \text { if } \quad k_{m, k}<0,\end{cases}
$$

where $k_{m, k}=k\left(t_{m}, x^{k}\right)$ and

$$
\begin{aligned}
& u_{x_{i}}^{-}=\frac{u\left(t_{m}, x^{k}\right)-u\left(t_{m}, x^{k}-\Delta x_{i} e_{i}\right)}{\Delta x_{i}}, \\
& u_{x_{i}}^{+}=\frac{u\left(t_{m}, x^{k}+\Delta x_{i} e_{i}\right)-u\left(t_{m}, x^{k}\right)}{\Delta x_{i}},
\end{aligned}
$$


$\Delta x_{i}$ is the discretization step in the spatial variable $x_{i}$, and $\left\{e_{i}=(0, \ldots, \underbrace{1}_{i}, \ldots, 0)\right\}_{i=1, \ldots, n}$ are the vectors of the standard basis. For the mean curvature operator we use the following discretization:

$$
\operatorname{div}\left(\frac{\nabla u}{|\nabla u|}\right)\left(t_{m}, x^{k}\right) \approx\left\{\begin{array}{ccc}
\sum_{i=1}^{n} u_{x_{i} x_{i}}-\sum_{i, j=1}^{n} \frac{u_{x_{i}} u_{x_{j}}}{\sum_{i=1}^{n}\left(u_{x_{i}}\right)^{2}} u_{x_{i} x_{j}} & \text { if } & \sum_{i=1}^{n}\left(u_{x_{i}}\right)^{2}>0 \\
0 & \text { otherwise }
\end{array}\right.
$$

where

$$
\begin{aligned}
u_{x_{i}} & =\frac{u\left(t_{m}, x^{k}+\Delta x_{i} e_{i}\right)-u\left(t_{m}, x^{k}-\Delta x_{i} e_{i}\right)}{2 \Delta x_{i}}, \\
u_{x_{i} x_{j}} & =\left\{\begin{array}{lrr}
\frac{u\left(t_{m}, x^{k}+\Delta x_{i} e_{i}\right)-2 u\left(t_{m}, x^{k}\right)+u\left(t_{m}, x^{k}-\Delta x_{i} e_{i}\right)}{\left(\Delta x_{i}\right)^{2}} & \text { if } & i=j, \\
\frac{\sum_{h_{1}, h_{2} \in\{-1,1\}} h_{1} h_{2} u\left(t_{m}, x^{k}+h_{1} \Delta x_{i} e_{i}+h_{2} \Delta x_{j} e_{j}\right)}{4 \Delta x_{i} \Delta x_{j}} & \text { otherwise. }
\end{array}\right.
\end{aligned}
$$

By combining the discretization of the forcing term and the mean curvature term we obtain the following explicit discretization scheme for the full PDE:

$$
\frac{u\left(t_{m+1}, x^{k}\right)-u\left(t_{m}, x^{k}\right)}{\Delta t}=\operatorname{div}\left(\frac{\nabla u}{|\nabla u|}\right)\left(t_{m}, x^{k}\right)+k_{m, k}|\nabla u|\left(t_{m}, x^{k}\right) .
$$

In the image boundary we assume the usual homogeneous Neumann boundary condition. From the above numerical scheme, we observe that $u\left(t_{m+1}, x^{k}\right)$ is computed as a nonlinear combination of $\left\{u\left(t_{m}, x^{k^{\prime}}\right)\right\}_{k^{\prime} \in N^{\prime}}$, where $\left\{x^{k^{\prime}}\right\}_{k^{\prime} \in N^{\prime}}$ represents a neighborhood of $x^{k}$. We are going to fix a CFL condition so that the diffusion coefficient associated to the term $u\left(t_{m}, x^{k}\right)$ is positive. First, in the case of the forcing term, we take into account that if we denote by

$$
u_{\max }=\left\{u\left(t_{m}, x^{k^{\prime \prime}}\right): k^{\prime \prime} \in N^{\prime}:\left|u\left(t_{m}, x^{k^{\prime}}\right)-u\left(t_{m}, x^{k}\right)\right| \leq\left|u\left(t_{m}, x^{k^{\prime \prime}}\right)-u\left(t_{m}, x^{k}\right)\right| \quad \forall k^{\prime} \in N^{\prime}\right\},
$$

then

$$
\sqrt{\sum_{i=1}^{n}\left(\max \left\{\max \left\{u_{x_{i}}^{+}, 0\right\}, \min \left\{u_{x_{i}}^{-}, 0\right\}\right\}\right)^{2}} \leq\left|u\left(t_{m}, x^{k}\right)-u_{\max }\right| \sqrt{\sum_{i=1}^{n} \frac{1}{\left(\Delta x_{i}\right)^{2}}} ;
$$

on the other hand, in the case of the mean curvature operator the absolute value of the diffusion coefficient associated to $u\left(t_{m}, x^{k}\right)$ is given by

$$
\sum_{i=1}^{n} \frac{2}{\left(\Delta x_{i}\right)^{2}}-\sum_{i=1}^{n} \frac{\left(u_{x_{i}}\right)^{2}}{\sum_{i=1}^{n}\left(u_{x_{i}}\right)^{2}} \frac{2}{\left(\Delta x_{i}\right)^{2}} \geq \sum_{i=1}^{n} \frac{-2}{\left(\Delta x_{i}\right)^{2}}+\frac{2}{\min _{i}\left(\Delta x_{i}\right)^{2}}>0 .
$$

Therefore, based on these expressions we propose the following CFL type condition for the choice of the time step $\Delta t$ :

$$
\Delta t\left(\sum_{i=1}^{n} \frac{2}{\left(\Delta x_{i}\right)^{2}}-\frac{2}{\min _{i}\left(\Delta x_{i}\right)^{2}}+\|k\|_{\infty} \sqrt{\sum_{i=1}^{n} \frac{1}{\left(\Delta x_{i}\right)^{2}}}\right)<1
$$


we point out that this CFL type condition does not guarantee the $L^{\infty}$ stability of the scheme. It is well known that in contrast with the solution of the continuous problem, in the discrete case, the explicit finite difference scheme for the mean curvature operator does not preserve the $L^{\infty}$ norm. So, as proposed in [1], we force a local $L^{\infty}$ norm preservation condition by introducing the following modification in the scheme:

$$
u\left(t_{n+1}, x_{k}\right)=\mathbf{T}_{m(u, n, k)}^{M(u, n, k)}\left(u\left(t_{n}, x_{k}\right)+\Delta t\left(\operatorname{div}\left(\frac{\nabla u}{|\nabla u|}\right)|\nabla u|+k|\nabla u|\right)\left(t_{n}, x_{k}\right)\right),
$$

where $m(u, n, k)=\min _{B_{1}\left(x_{k}\right)} u\left(t_{n}, x\right), M(u, n, k)=\max _{B_{1}\left(x_{k}\right)} u\left(t_{n}, x\right), B_{1}\left(x_{k}\right)$ is the ball centered in $x_{k}$ with radius 1 , and $\mathbf{T}_{a}^{b}(s)$ is the truncation function

$$
\mathbf{T}_{a}^{b}(s)=\left\{\begin{array}{ccc}
a & \text { if } & s<a \\
s & \text { if } & a \leq s \leq b \\
b & \text { if } & s>b
\end{array}\right.
$$

For simplicity in the exposition we choose the radius equal to 1 but, of course, we can use a radius bigger than 1 .

We choose as initial datum $u_{0}(x)$ an approximation of the signed distance function $d_{U_{0}, \beta}($.$) .$ To compute such approximation we use the following fast scheme based on the morphological operators erosion, $E_{t}\left(U_{0}\right)$, and dilation, $D_{t}\left(U_{0}\right)$, using a ball of radius $t$ as structuring element:

$$
d_{U_{0}, \beta}(x) \approx\left\{\begin{array}{cll}
-\sup \left\{t: x \in E_{t}\left(U_{0}\right)\right\} & \text { if } & x \in U_{0} \\
\inf \left\{\beta, \inf \left\{t: x \in D_{t}\left(U_{0}\right)\right\}\right\} & \text { if } & x \notin U_{0}
\end{array}\right.
$$

We use $t \in N$ and $E_{t}\left(U_{0}\right), D_{t}\left(U_{0}\right)$ are estimated by iterations of erosion and dilations computed with $t=1$, that is, we use that

$$
E_{t}\left(U_{0}\right)=\underbrace{E_{1} \circ \cdots \circ E_{1}}_{t}\left(U_{0}\right), \quad D_{t}\left(U_{0}\right)=\underbrace{D_{1} \circ \cdots \circ D_{1}}_{t}\left(U_{0}\right) .
$$

In general, as explained in [26], the distance function is usually computed only in a neighborhood of $\partial U_{0}$ and recomputed after a number of iterations. However, in this paper we do not recompute the distance function because we are interested in the long time behavior of the solution $u(t, x)$ of $(1)$.

7. Application of (1) to level set regularization. Let $U_{0} \subset \mathbf{R}^{n}$ be an open bounded set and let $h(x)$ be a Lipschitz bounded function satisfying

$$
U_{0}=\left\{x \in \mathbf{R}^{n}: h(x)<0\right\} .
$$

We are going to choose $k(x)$ in the following way:

$$
k_{\alpha, \gamma}(x)=\phi_{\alpha, \gamma}(h(x)),
$$

where $\phi_{\alpha, \gamma}: \mathbf{R} \rightarrow \mathbf{R}$ is the increasing function given by

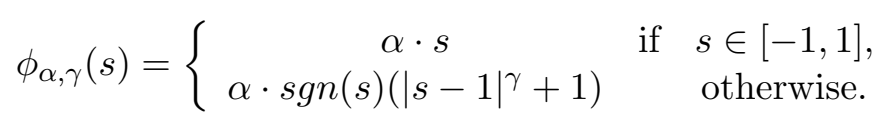


The parameter $\alpha>0$ is introduced to balance the mean curvature term and the forcing term. Here $\operatorname{sgn}($.$) denotes the sign function. Parameter \gamma>0$ represents the Hölder continuity coefficient of the forcing term when $|h(x)|>1$. Taking into account Remark 5 we observe that in the points where $k(x)$ is nonnegative (that is, in $\mathbf{R}^{n}-U_{0}$ ), $U_{t}$ tends to shrink, and in the case $k(x)$ is negative (that is, in $U_{0}$ ), $U_{t}$ tends to expand if the mean curvature in such point is small enough, and otherwise it tends to shrink. In this way $U_{t}$ tends to regularize the boundary of $U_{0}$ and it also removes the connected components of $U_{0}$ with a small size. Following the type of problem we deal with, there are different ways to choose function $h(x)$. A basic choice is to take $h(x)=d_{U_{0}, \beta}(x)$ and then $k_{\alpha, \gamma}(x)$ given by

$$
k_{\alpha, \gamma}^{1}(x)=\phi_{\alpha, \gamma}\left(d_{U_{0}, \beta}(x)\right) .
$$

We point out that, in radial coordinates, if $U_{0}=B_{r_{0}}\left(x_{0}\right)$ and if we choose as forcing term $k_{\alpha, \gamma}^{1}(x)$, with $\gamma=1$, then $U_{t}=B_{r(t)}\left(x_{0}\right)$, where $r(t)$ satisfies

$$
\left\{\begin{array}{c}
\frac{d r}{d t}(t)=-\frac{n-1}{r(t)}-\alpha\left(r(t)-r_{0}\right) \quad \text { for } \quad r \in\left[0, r_{0}+S\right), \\
r(0)=r_{0},
\end{array}\right.
$$

for some $S>0$. We observe that this is a particular case of the forcing term considered in Lemma 7 with $m=\alpha$ and $c=-\alpha r_{0}$. Then, using the result quoted in Lemma 7 we obtain the following.

Theorem 11. Let $U_{t}$ be the evolution of the set $U_{0}=B_{r_{0}}\left(x_{0}\right)$ under the action of the geometric flow (1)-(4) with $k(r)=\phi_{\alpha, 1}\left(d_{U_{0}, \beta}(x)\right)$. Then $U_{t}=B_{r(t)}\left(x_{0}\right)$, where $r(t)$ is a nonincreasing function satisfying that

$$
\lim _{t \rightarrow \infty} r(t)=\frac{1}{2} r_{0}+\frac{1}{2} \sqrt{r_{0}^{2}-4 \frac{n-1}{\alpha}}
$$

if $r_{0} \geq 2 \sqrt{\frac{n-1}{\alpha}}$ or

if $r_{0}<2 \sqrt{\frac{n-1}{\alpha}}$.

$U_{t}$ vanishes in a finite time,

Proof. It is a straightforward application of Lemma 7.

Remark 8. We point out that from the above theorem and Theorems 5 and 10 we obtain that if we use as forcing term $k(r)=\phi_{\alpha, 1}\left(d_{U_{0}, \beta}(x)\right)$ and if $U_{0}=\cup_{k} U_{0}^{k}$, where $\left\{U_{0}^{k}\right\}$ are connected sets such that there exist $B_{r_{0}^{k}}\left(x_{0}^{k}\right) \subseteq U_{0}^{k} \subseteq B_{r_{1}^{k}}\left(x_{1}^{k}\right)$ with distance $\left(B_{r_{1}^{k}}\left(x_{1}^{k}\right), B_{r_{1}^{k \prime}}\left(x_{1}^{k^{\prime}}\right)\right)>$ $\delta>0$ for $k \neq k^{\prime}$, then each $U_{0}^{k}$ evolves in an independent way $\forall t>0$ and satisfies that $B_{r_{0}^{k}(t)}\left(x_{0}^{k}\right) \subseteq U_{t}^{k} \subseteq B_{r_{1}^{k}(t)}\left(x_{1}^{k}\right)$, where $r_{0}^{k}(t)$ and $r_{1}^{k}(t)$ are the solutions of (36) for $r_{0}=r_{0}^{k}$ and $r_{0}=r_{1}^{k}$, respectively. In particular, if $r_{1}^{k}<2 \sqrt{\frac{n-1}{\alpha}}$, then $U_{t}^{k}$ vanishes in a finite time, and if

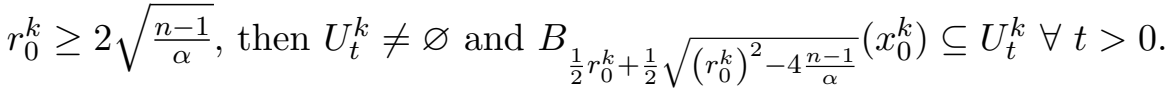

In the case in which $U_{0}$ is an image level set, that is, if $U_{0}=U_{0}^{c}=\left\{x \in \mathbf{R}^{n}: I(x)<c\right\}$, where $I: \mathbf{R}^{n} \rightarrow \mathbf{R}$ is an image, then we can choose $h(x)$ depending of the image intensity value. That is, we can choose $h(x)=I(x)-c$ and take as forcing term 


$$
k_{\alpha, \gamma}^{2}(x)=\phi_{\alpha, \gamma}(I(x)-c)
$$

We notice that in this case, the forcing term depends on the image contrast: the higher the level set contrast in the image, the closer it will be $U_{t}$ to $U_{0}$. As we will see later, in some applications we are interested in the regularization of the image level set $U_{0}=U_{0}^{c_{1}, c_{2}}=\{x \in$ $\left.\mathbf{R}^{n}: c_{1}<I(x)<c_{2}\right\}$. In such case we can choose

$$
k_{\alpha, \gamma}^{3}(x)=\phi_{\alpha, \gamma}\left(\left(I(x)-c_{1}\right)\left(I(x)-c_{2}\right)\right) .
$$

In Figure 6 we show a 2D example of set $U_{0}$, and the associated distance function $d_{U_{0}, \beta}(x)$, which we use to illustrate the shape of the set evolution $U_{t}$ under the action of the geometric flow (1)-(4). In Figures 7-15 we show the shape of $U_{t}$ for different choices of the forcing term $k(x)$. In each figure, we include a link to a video to show the evolution of $U_{t}$. We point out that in the case we choose as forcing term $k_{\alpha, \gamma}^{1}(x)$, the solution, $u(t, x)$, of (1)-(4) tends to a nontrivial asymptotic state. We have included in $U_{0}$ circles of different sizes and we observe, as predicted by the theoretical results, that according to the values of $\alpha$ and $\gamma$ and the circles size, the circles vanish in finite time or they remain in the set $U_{t}$.

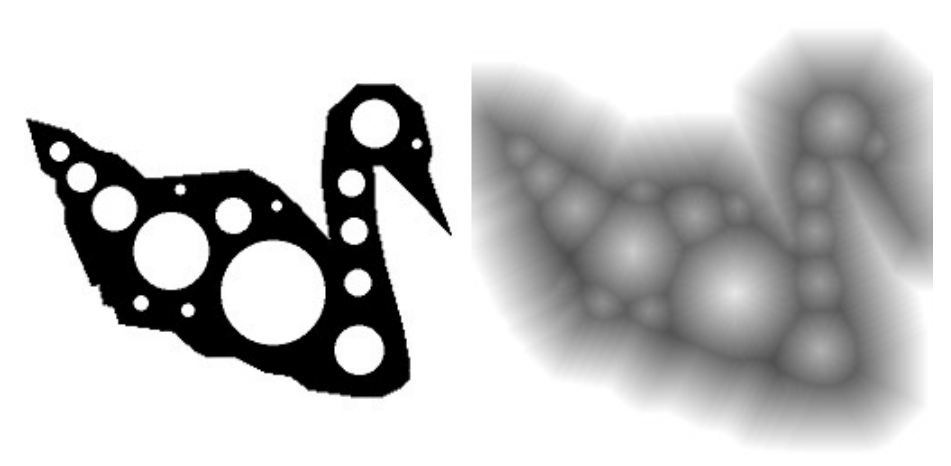

Figure 6. From left to right we show a silhouette (in black) used as initial set $U_{0}$ and the signed distance function $d_{U_{0}, \beta}(x)$ scaled between 0 and 255 for visualization purposes.
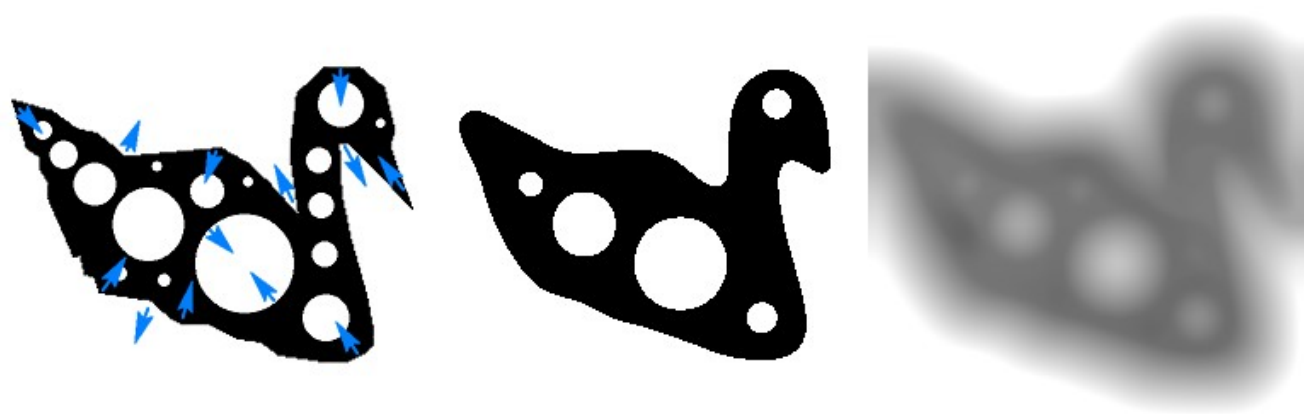

Figure 7. Evolution of $U_{t}$ for (1)-(4) without forcing term ( $\left.k \equiv 0\right)$. From left to right we show (i) the original set $U_{0}$ where we illustrate with blue arrows the initial motion direction of $\Gamma_{0}$, (ii) the set $U_{t}$ for $t=50$, and (iii) the solution $u(t, x)$ of (1)-(4) for $t=50$ (video with the evolution of $U_{t}$ and $u(t, x)$ ). 


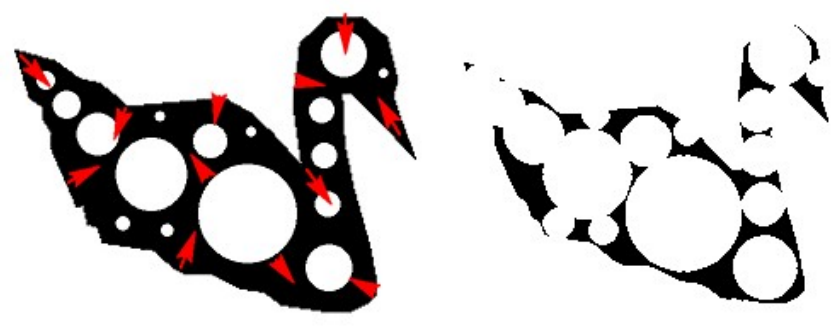

Figure 8. Evolution of $U_{t}$ for equation $\frac{\partial u}{\partial t}=0.1|D u|$. From left to write we show (i) the original set $U_{0}$ where we illustrate with red arrows the initial motion direction of $\Gamma_{0}$, (ii) the set $U_{t}$ for $t=50$, and (iii) the solution $u(t, x)$ of (1)-(4) for $t=50$ (video with the evolution of $U_{t}$ and $u(t, x)$ ).

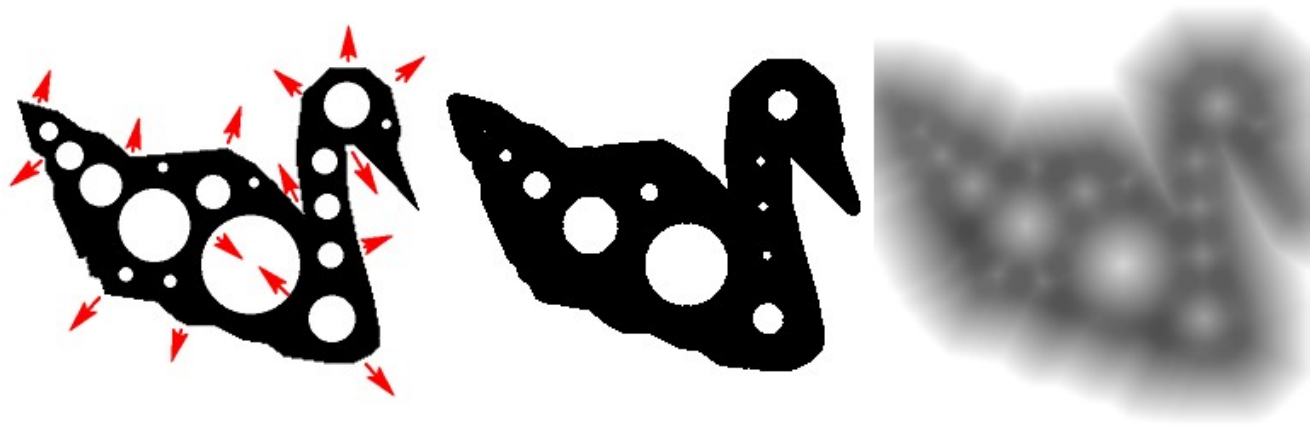

Figure 9. Evolution of $U_{t}$ for equation $\frac{\partial u}{\partial t}=-0.1|D u|$. From left to write we show (i) the original set $U_{0}$ where we illustrate with red arrows the initial motion direction of $\Gamma_{0}$, (ii) the set $U_{t}$ for $t=50$, and (iii) the solution $u(t, x)$ of (1)-(4) for $t=50$ (video with the evolution of $U_{t}$ and $u(t, x)$ ).
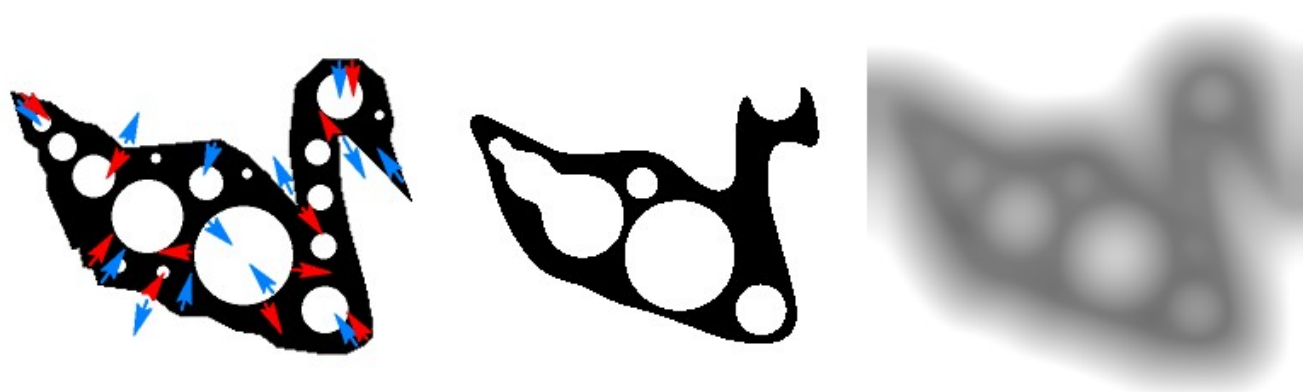

Figure 10. Evolution of $U_{t}$ for (1)-(4) using as forcing term $k(x) \equiv 0.1$. From left to right we show (i) the original set $U_{0}$ where we illustrate with blue arrows the initial motion direction with respect to the mean curvature term and with red arrows with respect to the forcing term, (ii) the set $U_{t}$ for $t=50$, and (iii) the solution $u(t, x)$ of (1)-(4) for $t=50$ (video with the evolution of $U_{t}$ and $u(t, x)$ ). 

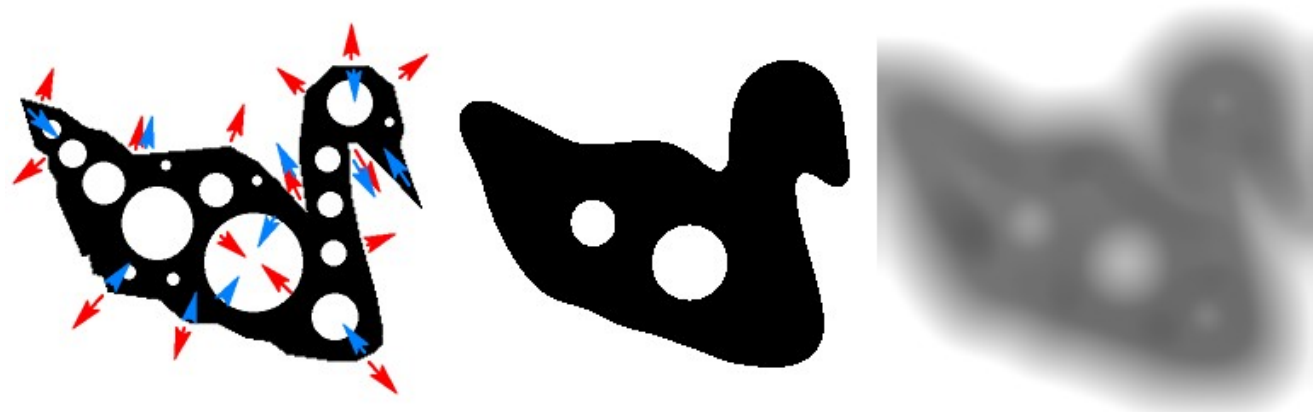

Figure 11. Evolution of $U_{t}$ for (1)-(4) using as forcing term $k(x) \equiv-0.1$. From left to right we show (i) the original set $U_{0}$ where we illustrate with blue arrows the initial motion direction with respect to the mean curvature term and with red arrows with respect to the forcing term, (ii) the set $U_{t}$ for $t=50$, and (iii) the solution $u(t, x)$ of (1)-(4) for $t=50$ (video with the evolution of $U_{t}$ and $u(t, x)$ ).
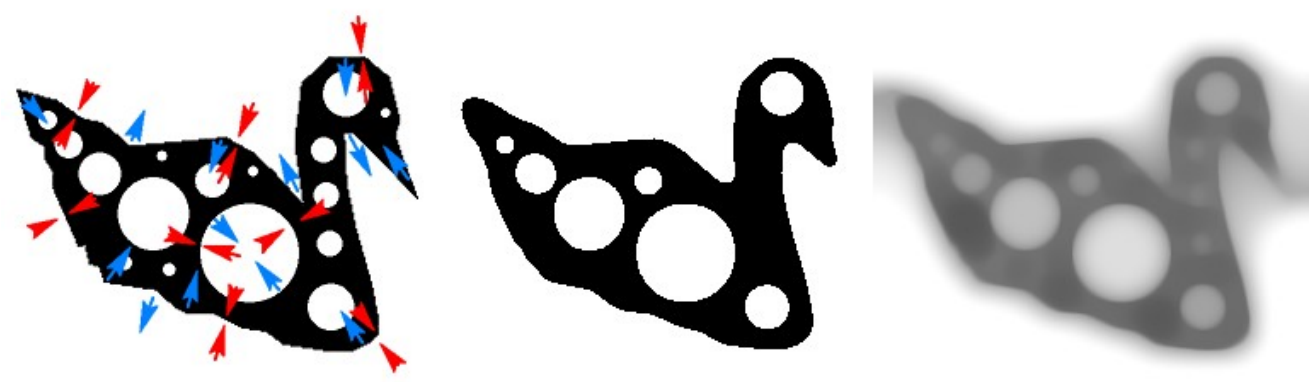

Figure 12. Evolution of $U_{t}$ for (1)-(4) using as forcing term $k_{\alpha, \gamma}^{1}(x)$ with $\alpha=0.1$ and $\gamma=0.5$. From left to right we show (i) the original set $U_{0}$ where we illustrate with blue arrows the initial motion direction with respect to the mean curvature term and with red arrows with respect to the forcing term, (ii) the set $U_{t}$ for $t=50$, and (iii) the solution $u(t, x)$ of (1)-(4) for $t=50$ (video with the evolution of $U_{t}$ and $u(t, x)$ ).
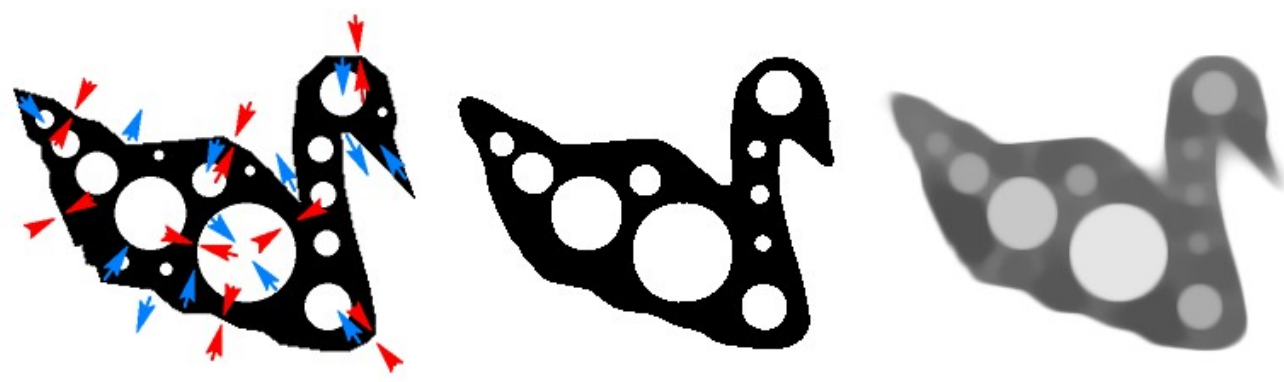

Figure 13. Evolution of $U_{t}$ for (1)-(4) using as forcing term $k_{\alpha, \gamma}^{1}(x)$ with $\alpha=0.1$ and $\gamma=1$. From left to right we show (i) the original set $U_{0}$ where we illustrate with blue arrows the initial motion direction with respect to the mean curvature term and with red arrows with respect to the forcing term, (ii) the set $U_{t}$ for $t=50$, and (iii) the solution $u(t, x)$ of (1)-(4) for $t=50$ (video with the evolution of $U_{t}$ and $u(t, x)$ ). 


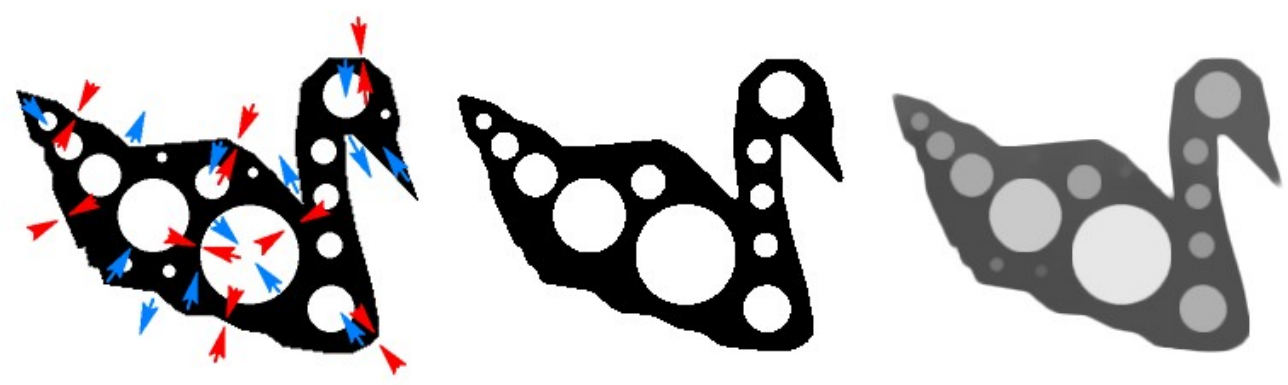

Figure 14. Evolution of $U_{t}$ for (1)-(4) using as forcing term $k_{\alpha, \gamma}^{1}(x)$ with $\alpha=0.5$ and $\gamma=1$. From left to right we show (i) the original set $U_{0}$ where we illustrate with blue arrows the initial motion direction with respect to the mean curvature term and with red arrows with respect to the forcing term, (ii) the set $U_{t}$ for $t=50$, and (iii) the solution $u(t, x)$ of (1)-(4) for $t=50$ (video with the evolution of $U_{t}$ and $u(t, x)$ ).
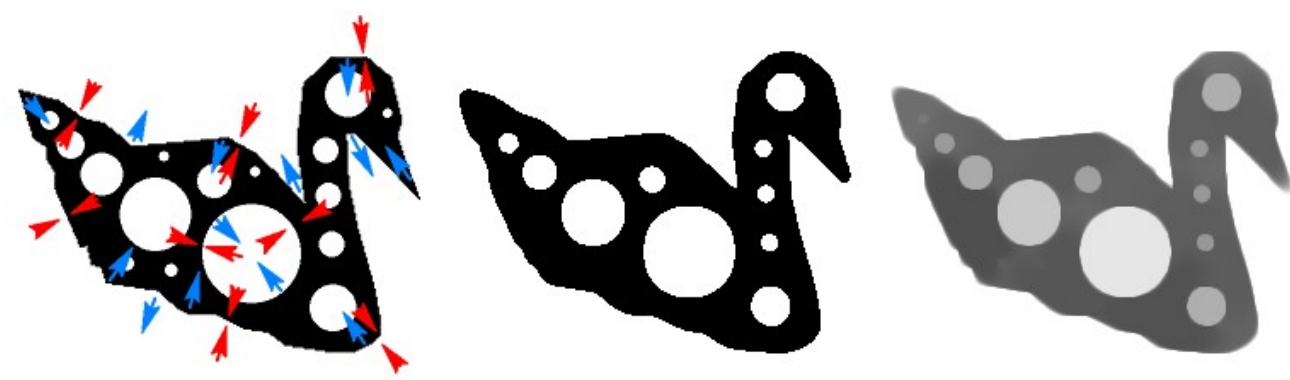

Figure 15. Evolution of $U_{t}$ for (1)-(4) using as forcing term $k(x)=k_{R_{0}, \gamma, C}\left(d_{U_{0}, \beta}(x)+R_{0}\right)$, where $k_{R_{0}, \gamma, C}($. is given by (32) and $R_{0}=3, \gamma=0.5$, and $C=1$. From left to right we show (i) the original set $U_{0}$ where we illustrate with blue arrows the initial motion direction with respect to the mean curvature term and with red arrows with respect to the forcing term, (ii) the set $U_{t}$ for $t=50$, and (iii) the solution $u(t, x)$ of (1)-(4) for $t=50$ (video with the evolution of $U_{t}$ and $u(t, x)$ ).

Next we present an experiment using the real 2D image, $I: \Omega \subset \mathbf{R}^{2} \rightarrow\{0,1,2, . ., 255\}$, shown in Figure 16(a). In this figure, we also show some level sets $U_{0}^{c}=\{x: I(x) \leq c\}$ for different values of levels $c$. Using the approach presented in [12], we consider the image topographic map, that is, the collection of all its level sets $\left\{U_{0}^{c}\right\}_{c=0,1, . ., 255}$. Then, for each level $c$, we compute the level set evolution $U_{t}^{c}=\{x: u(t, x) \leq c\}$, where $u(t, x)$ is the solution of (1)-(4). Then, for each time $t$ we can recover a gray-level image $I_{t}$ using the expression

$$
I_{t}(x)=\inf \left\{c: x \in U_{t}^{c}\right\} \text {. }
$$

In Figure 17, we show, for the level set $U_{0}^{144}=\{x: I(x) \leq 144\}$, the distance function $d_{U_{0}^{144, \beta}}(x)$ and the solution $u(t, x)$ of (1)-(4) for $t=10$ and different choices of the forcing term $k(x)$. In Figure 18 we present the evolution of $U_{t}^{144}$ for $t=10$ and in Figure 19 the reconstruction of the image from the level sets using (39). 


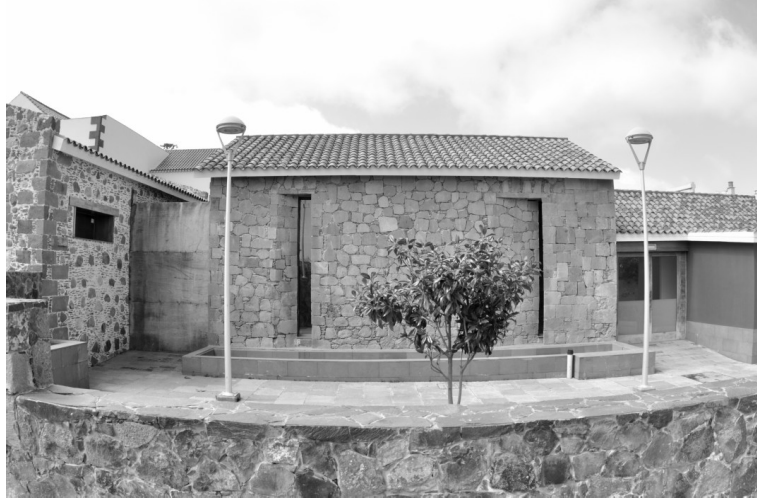

(a) Original image

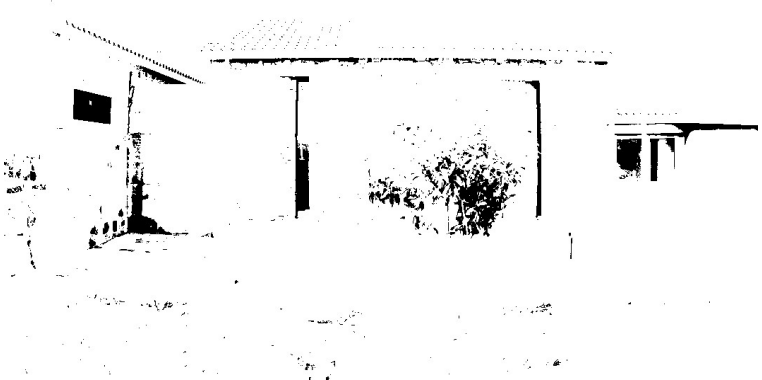

(b) level set $U_{0}^{58}=\{x: I(x) \leq 58\}$

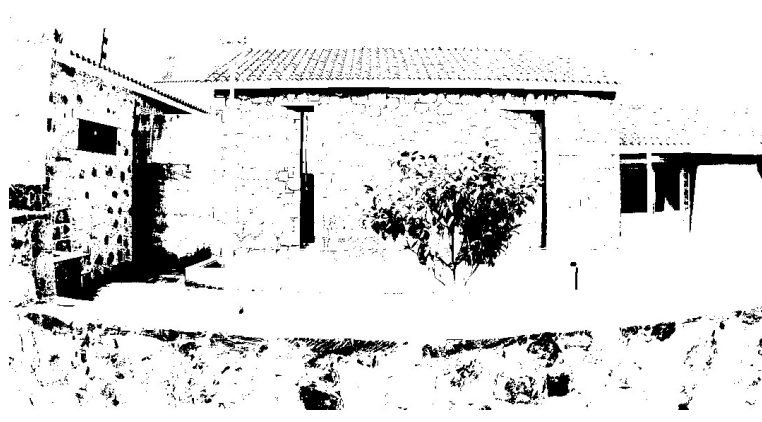

(d) level set $U_{0}^{94}=\{x: I(x) \leq 94\}$

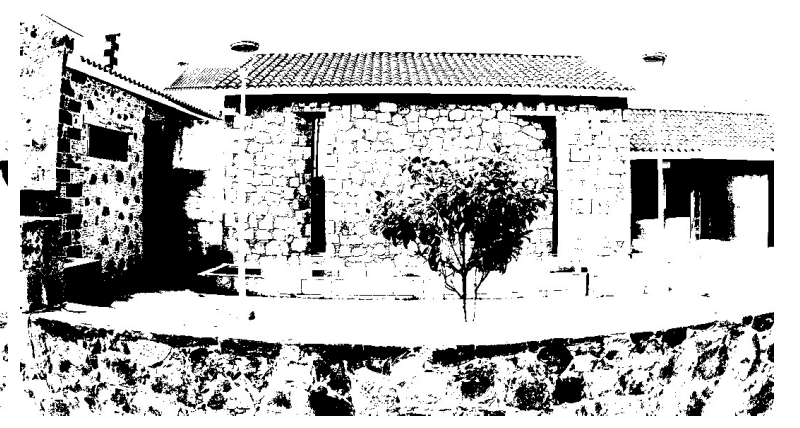

(e) level set $U_{0}^{121}=\{x: I(x) \leq 121\}$

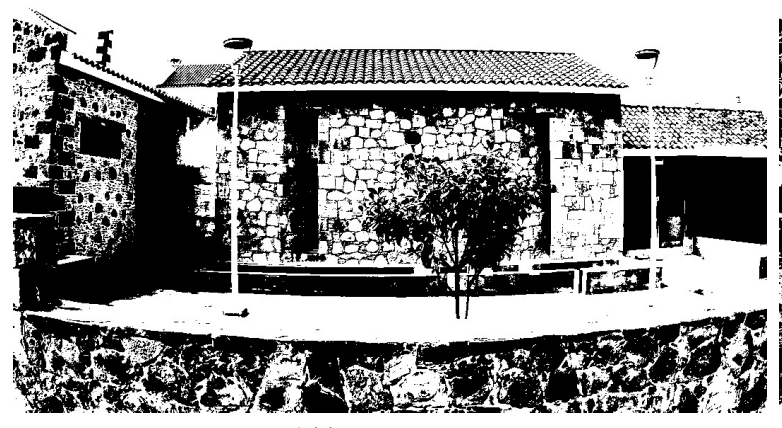

(e) level set $U_{0}^{144}=\{x: I(x) \leq 144\}$

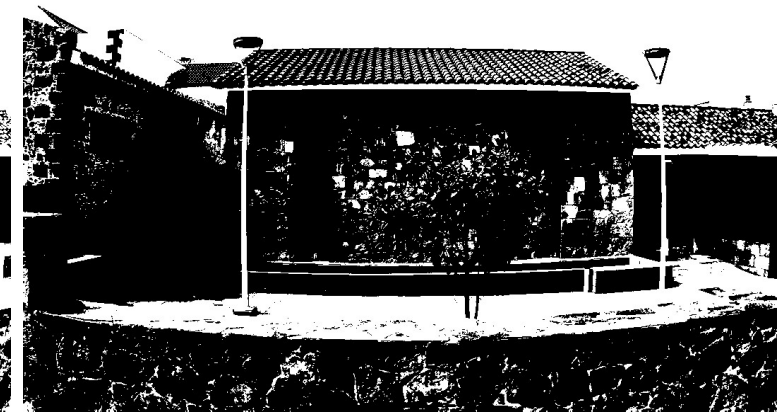

(f) level set $U_{0}^{175}=\{x: I(x) \leq 175\}$

Figure 16. We show a real image used in the experiments and some of its level sets $U_{0}^{c}=\{x: I(x) \leq c\}$ for different values of levels.

Next, we present an experiment on a real 3D image in the context of medical image segmentation. We use a CT (computed tomography) 3D scan, $I(x)$ (shown in Figure 21), of a patient suffering from severe aortic elongations. For medical diagnosis purposes, a segmentation of the aortic lumen is required. Usually, an initial segmentation is obtained by 


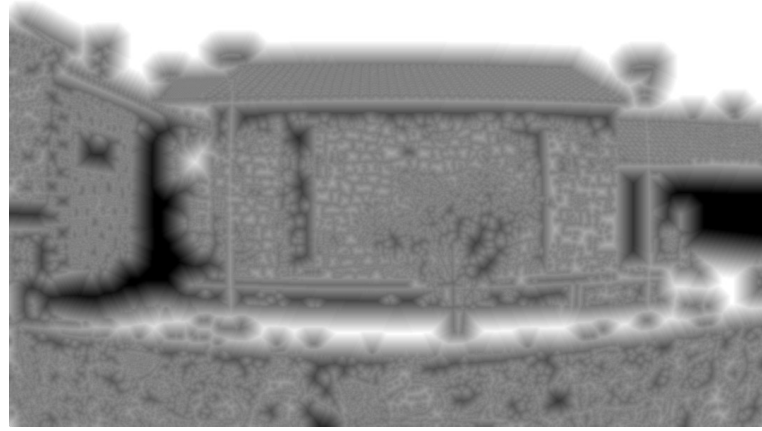

(a) distance function for the level set $U_{0}^{144}$

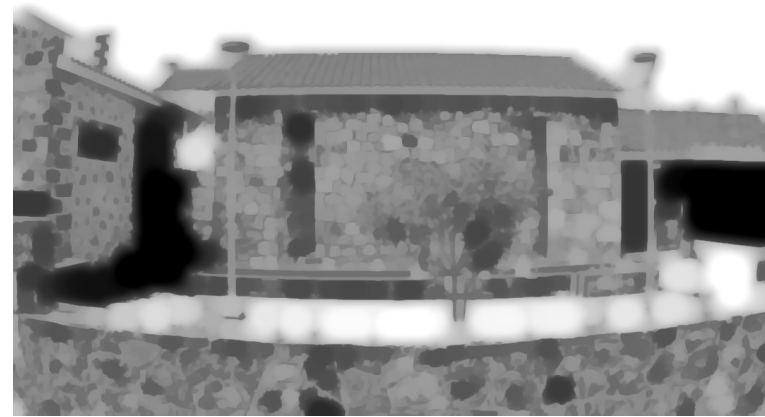

(c) $\frac{\partial u}{\partial t}=\operatorname{div}\left(\frac{\nabla u}{|\nabla u|}\right)|\nabla u|+k_{1,0.2}^{1}(x)|\nabla u|$

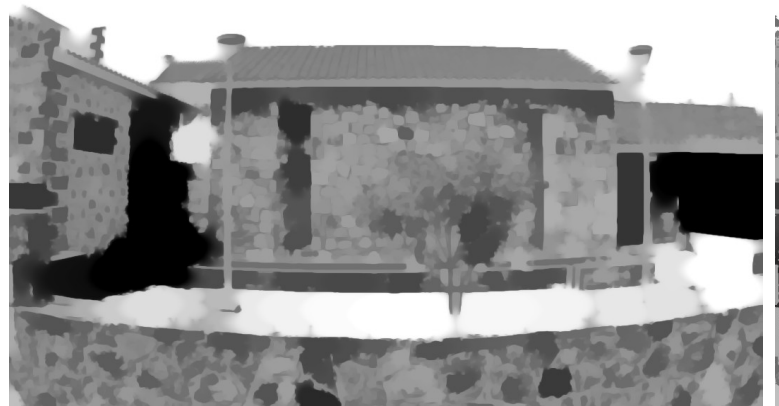

(e) $\frac{\partial u}{\partial t}=\operatorname{div}\left(\frac{\nabla u}{|\nabla u|}\right)|\nabla u|+k_{1,0.5}^{1}(x)|\nabla u|$

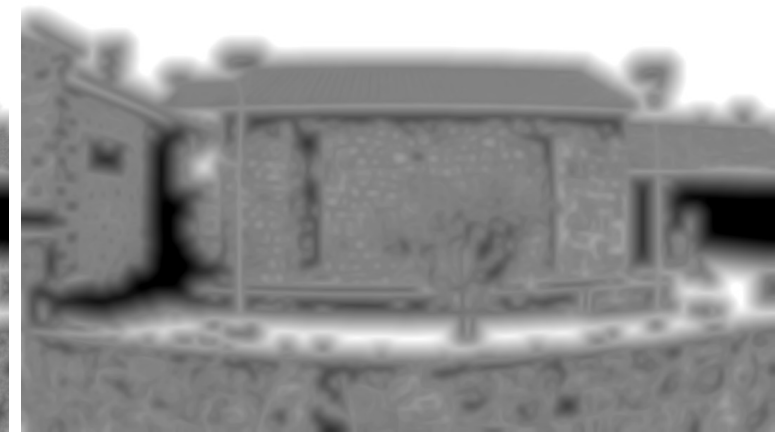

(b) regularization with $\frac{\partial u}{\partial t}=\operatorname{div}\left(\frac{\nabla u}{|\nabla u|}\right)|\nabla u|$

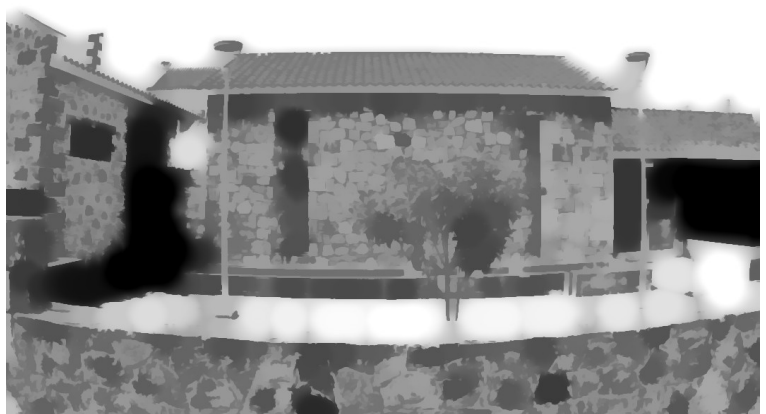

(d) $\frac{\partial u}{\partial t}=\operatorname{div}\left(\frac{\nabla u}{|\nabla u|}\right)|\nabla u|+k_{1,0.2}^{2}(x)|\nabla u|$

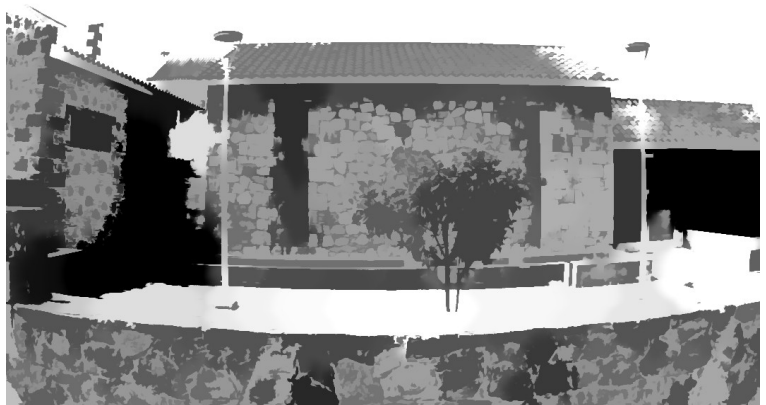

(f) $\frac{\partial u}{\partial t}=\operatorname{div}\left(\frac{\nabla u}{|\nabla u|}\right)|\nabla u|+k_{1,0.2}^{2}(x)|\nabla u|$

Figure 17. (a) Distance function of the real image shown in Figure 16 for the level set $U_{0}^{144}$. (b)-(f) Evolution of the distance function $d_{U_{0}^{144}, \beta}(x)$ under the action of (1)-(4) for $t=10$ and different choices of the forcing term $k(x)$.

thresholding the intensity value of the $\mathrm{CT}$ image between two values $c_{1}$ and $c_{2}$, that is, the set $U_{0}=\left\{x: c_{1}<I(x)<c_{2}\right\}$ is an initial segmentation of the aortic lumen. However (as shown in Figure 21), this initial segmentation is inaccurate because other human organs in 


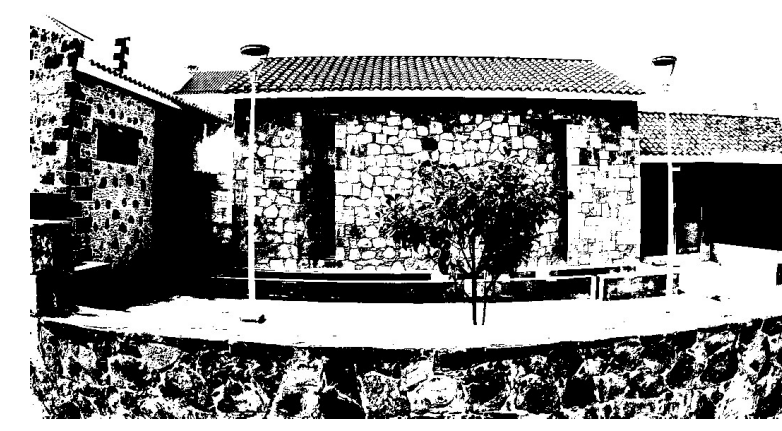

(a) level set $U_{0}^{144}$

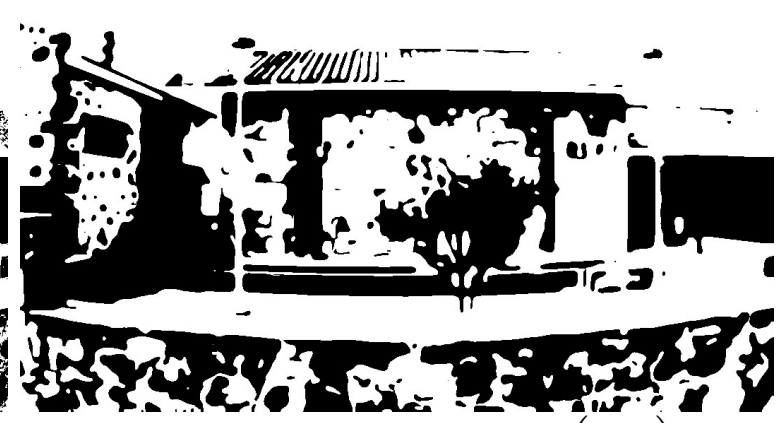

(b) regularization with $\frac{\partial u}{\partial t}=\operatorname{div}\left(\frac{\nabla u}{|\nabla u|}\right)|\nabla u|$

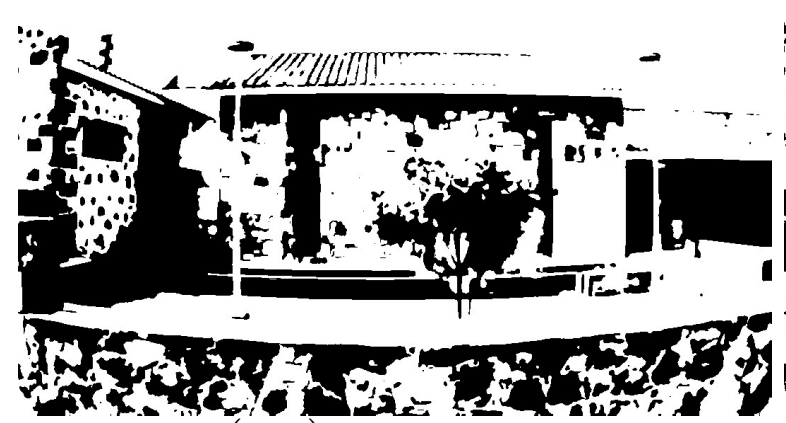

(c) $\frac{\partial u}{\partial t}=\operatorname{div}\left(\frac{\nabla u}{|\nabla u|}\right)|\nabla u|+k_{1,0.2}^{1}(x)|\nabla u|$
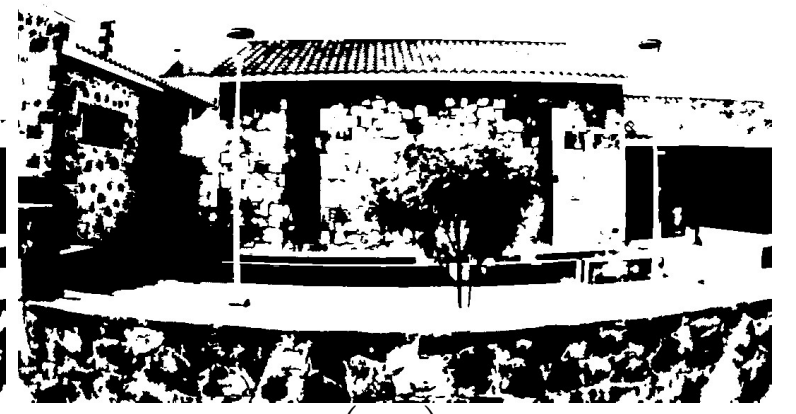

(d) $\frac{\partial u}{\partial t}=\operatorname{div}\left(\frac{\nabla u}{|\nabla u|}\right)|\nabla u|+k_{1,0.2}^{2}(x)|\nabla u|$

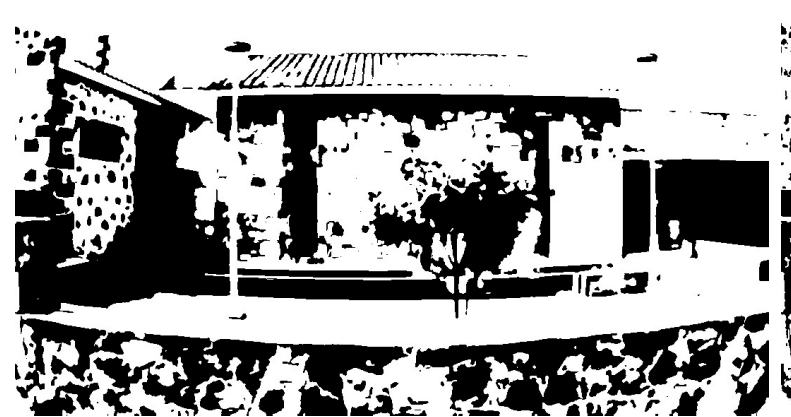

(e) $\frac{\partial u}{\partial t}=\operatorname{div}\left(\frac{\nabla u}{|\nabla u|}\right)|\nabla u|+k_{1,0.5}^{1}(x)|\nabla u|$
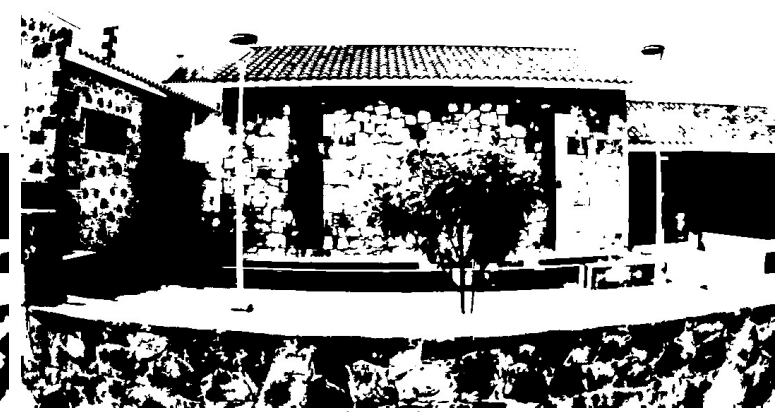

(f) $\frac{\partial u}{\partial t}=\operatorname{div}\left(\frac{\nabla u}{|\nabla u|}\right)|\nabla u|+k_{1,0.2}^{2}(x)|\nabla u|$

Figure 18. (a) level set $U_{0}^{144}$ of the real image shown in Figure 16. (b)-(f) Regularization of the level set $U_{0}^{144}$ under the action of (1)-(4) for $t=10$ and different choices of the forcing term $k(x)$.

the CT image have similar intensity values and we cannot fix thresholds $c_{1}$ and $c_{2}$ such that $U_{0}$ contains properly just the aorta lumen. Therefore some kind of processing is required to improve the quality of the initial estimation of $U_{0}$. We are going to use (1)-(4) to regularize the set $U_{0}$ and provide a better segmentation of the aortic lumen. In Figure 20 we show a 3D 


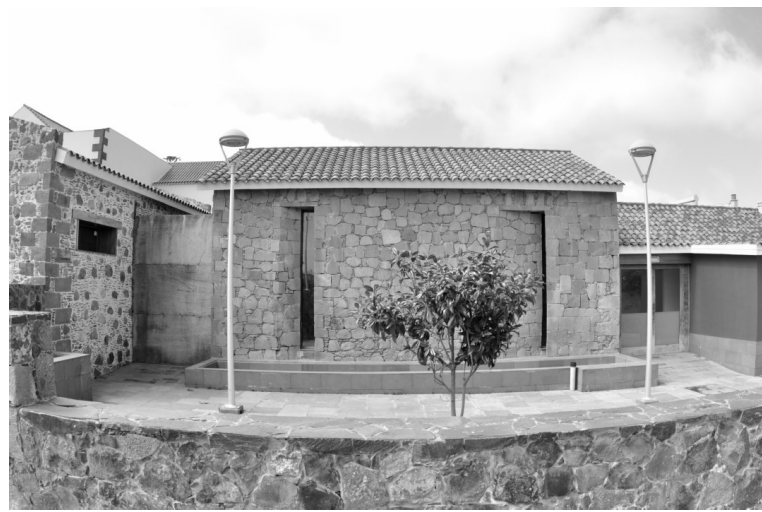

(a) Original image

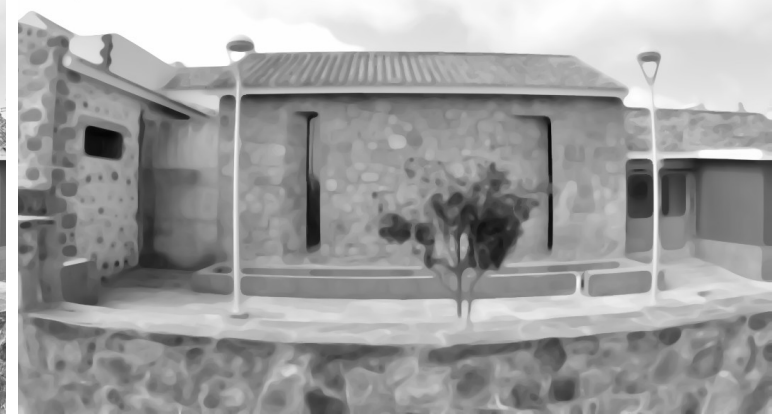

(b) regularization with $\frac{\partial u}{\partial t}=\operatorname{div}\left(\frac{\nabla u}{|\nabla u|}\right)|\nabla u|$

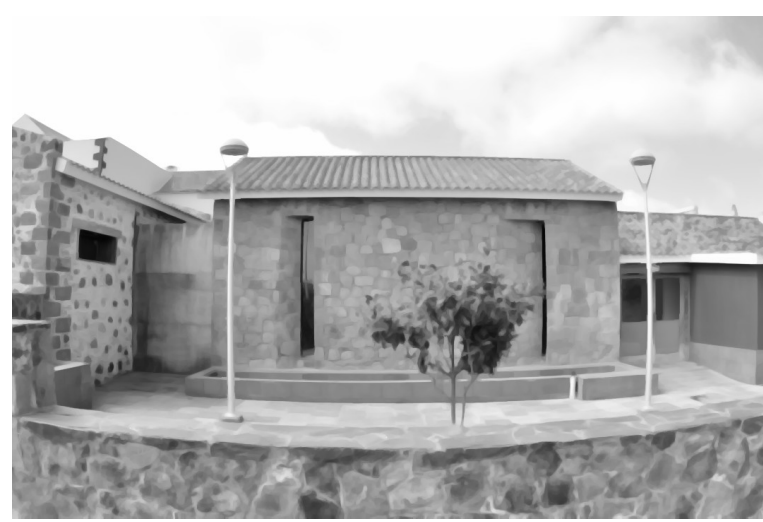

(c) $\frac{\partial u}{\partial t}=\operatorname{div}\left(\frac{\nabla u}{|\nabla u|}\right)|\nabla u|+k_{1,0.2}^{1}(x)|\nabla u|$
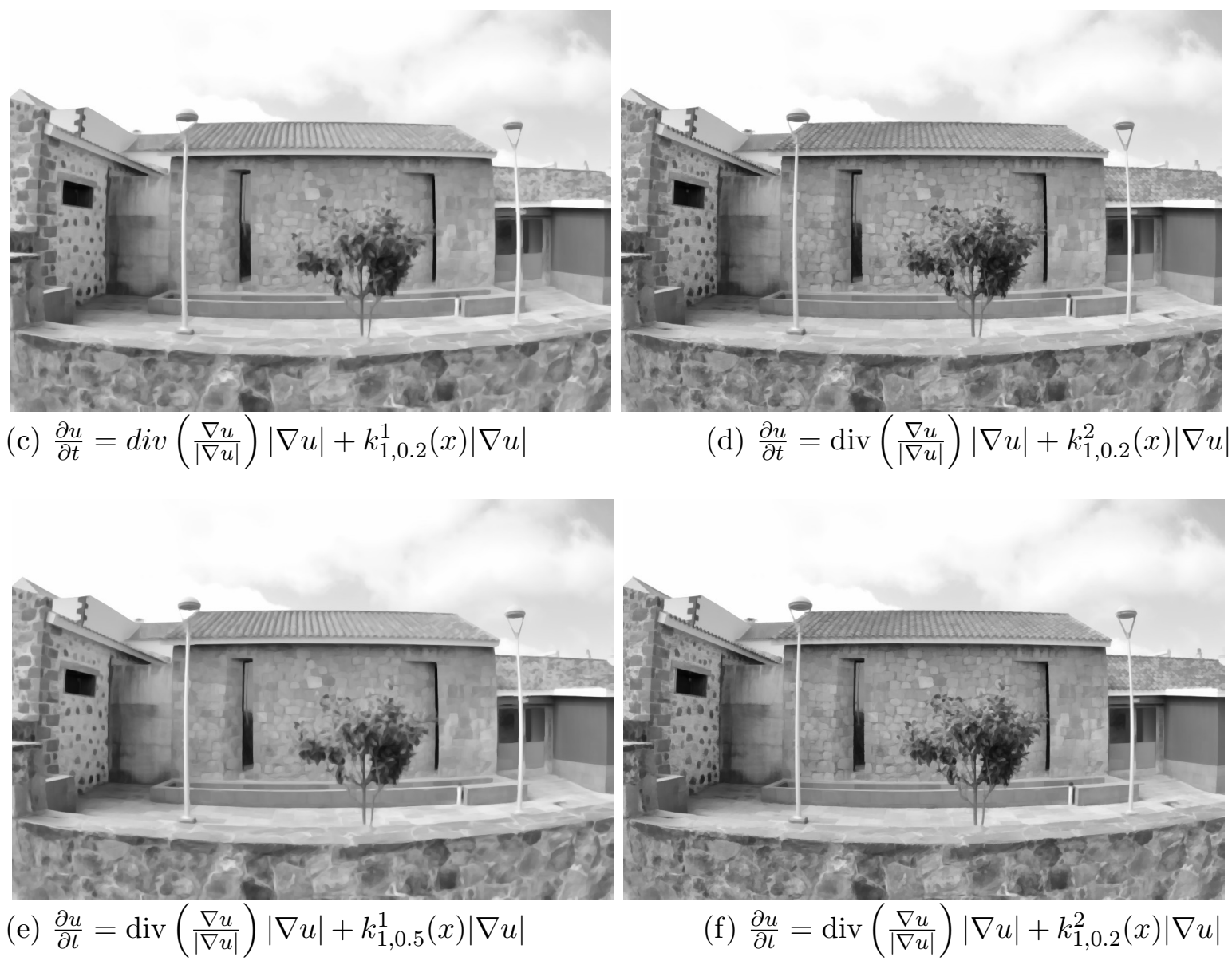

Figure 19. (a) Real image shown in Figure 16. (b)-(f) Reconstruction of the image from the level sets (using (39)) after regularization of the level sets using (1)-(4) for $t=10$ and different choices of the forcing term $k(x)$. 

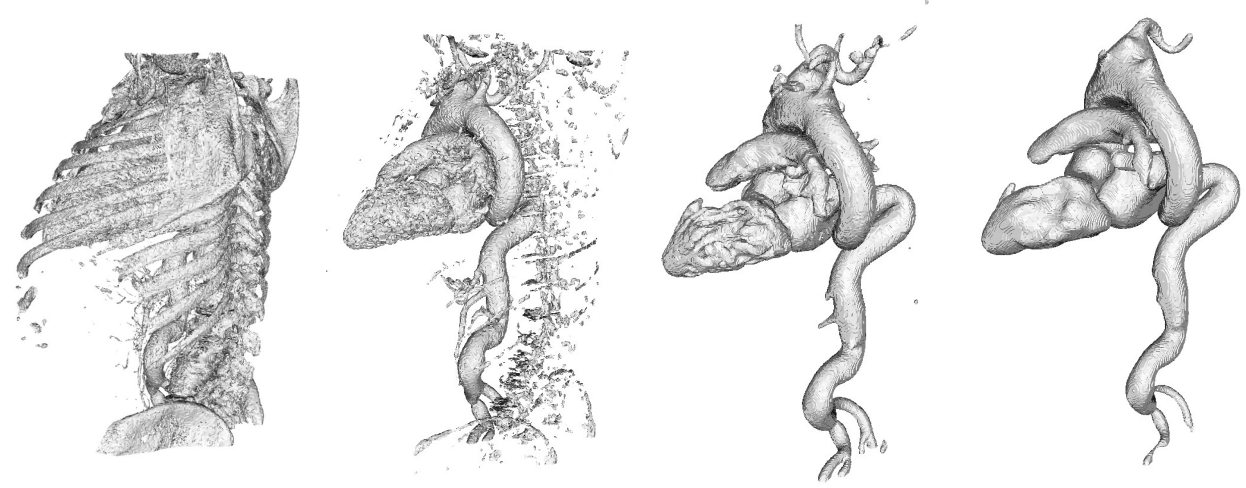

Figure 20. From left to right we show the level set $U_{0}$ and $U_{t}$ for $t=10$ using (1)-(4) with the forcing terms, $k_{1,0.2}^{3}(x), k_{0.1,0.2}^{3}(x)$, and $k \equiv 0$, respectively.
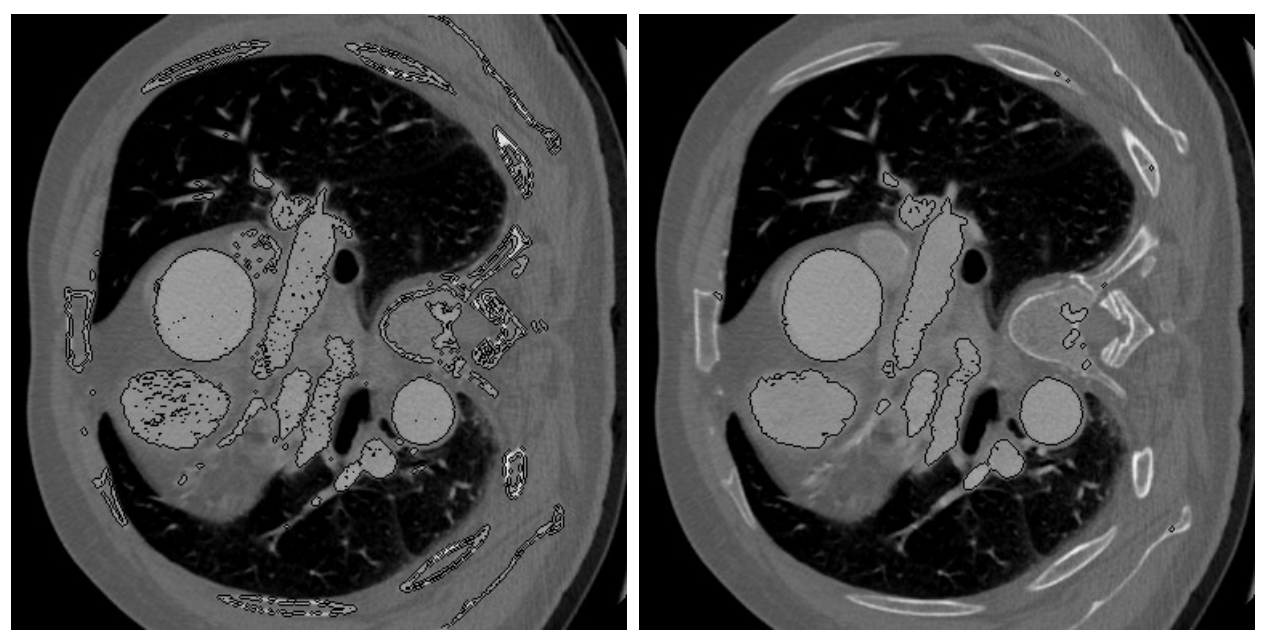

(a) original image and $\Gamma_{0}$ (in black) (video)

(b) $\Gamma_{10}$ for $k(x)=k_{1 ., 0.2}^{3}(x)$ (video)

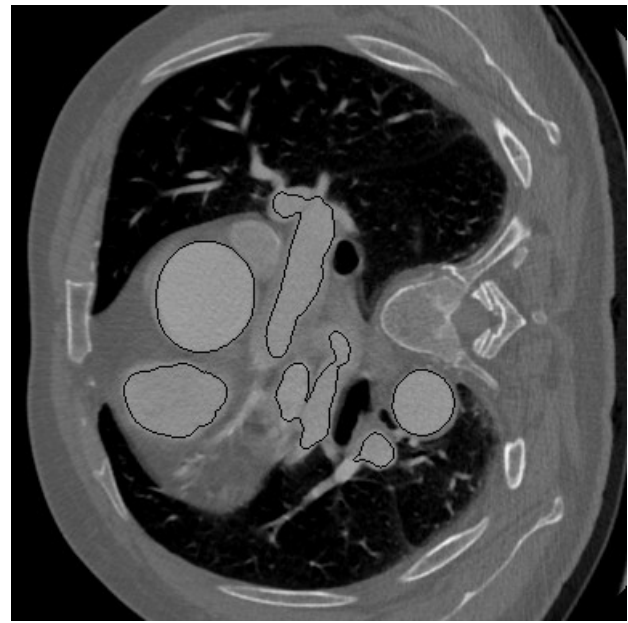

(c) $\Gamma_{10}$ for $k(x)=k_{0.1,0.2}^{3}(x)$ (video)

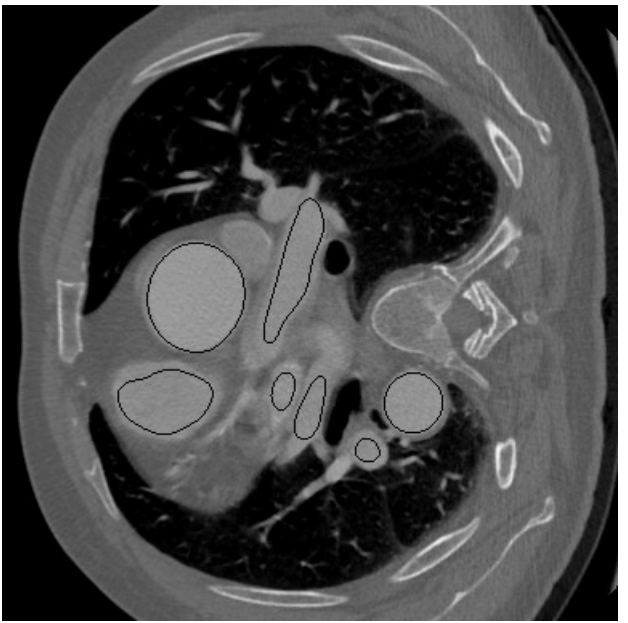

(d) $\Gamma_{10}$ for $k(x) \equiv 0$ (video)

Figure 21. We show one slice of the original image and $\Gamma_{t}=\partial U_{t}$ (in black) using (1)-(4) with different forcing terms. For each image we also include a link to a video with the full $3 D$ image. 

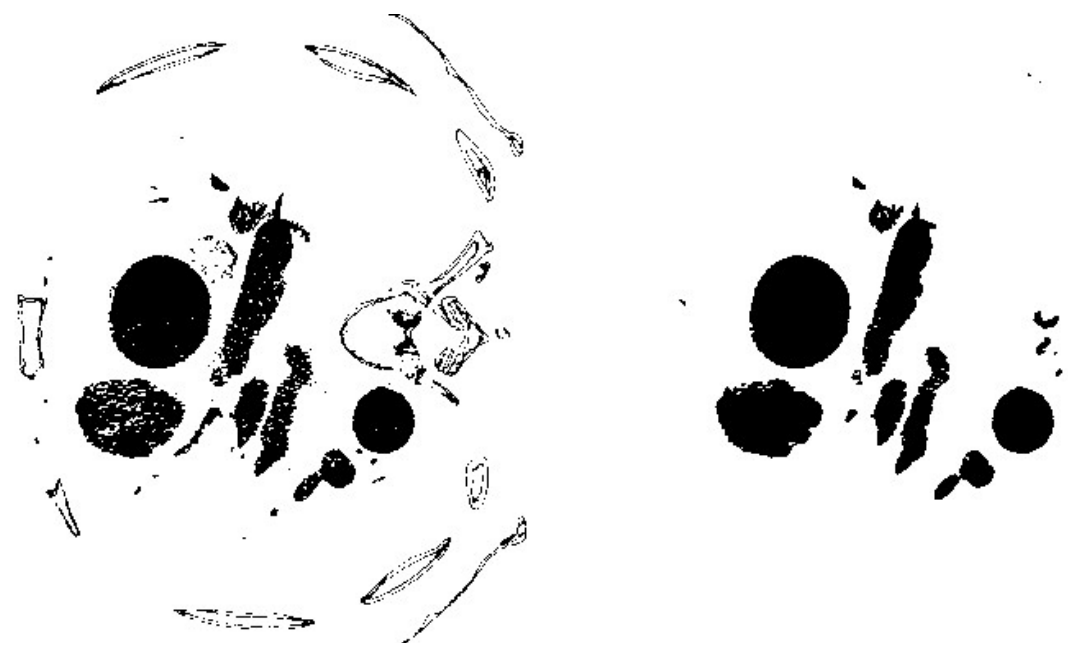

(a) $U_{0}=\left\{x: c_{1}<I(x)<c_{2}\right\}$ (video)

(b) $U_{10}$ using (1)-(4) for $k_{1,0.2}^{3}(x)$ (video)
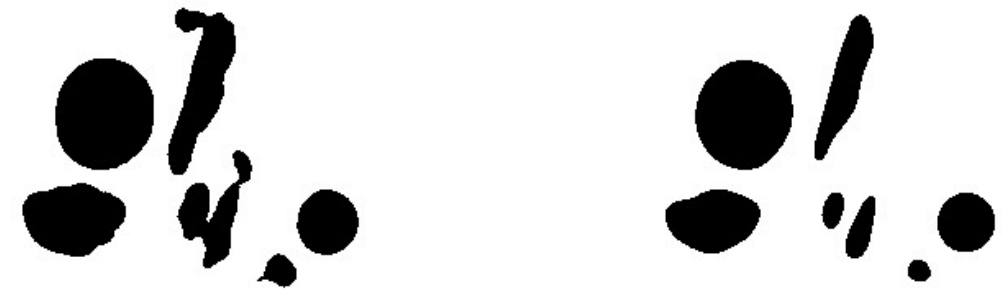

(c) $U_{10}$ using (1)-(4) for $k_{0.1,0.2}^{3}(x)$ (video)

(d) $U_{10}$ using (1)-(4) for $k(x) \equiv 0$ (video)

Figure 22. We show one slice of the level set $U_{0}=\left\{x: c_{1}<I(x)<c_{2}\right\}$ as well as $U_{t}=\{x: u(10, x) \leq 0\}$ where $u(t, x)$ is the solution of (1)-(4) for $t=10$ with different forcing terms. For each image we also include a link to a video with the full $3 D$ sequence.

representation of the sets $U_{t}$ for different forcing terms. We use as the forcing term $k(x)$, the one given in (38). In Figure 21 we show $\Gamma_{0}=\partial U_{0}$ the initial contours as well as $\Gamma_{t}=\partial U_{t}$ for different choices of the forcing terms. We observe that by adjusting the parameters $\alpha$ and $\gamma$ in the forcing term (38) we obtain different degrees of regularization; we point out that in the case of no forcing term $(k \equiv 0)$ we regularize too much and the contour $\Gamma_{t}$ tends to move away from the aorta lumen contour actual location. In Figures 22 and 23 we show the level set and distances associated to the different forcing terms.

8. Conclusions. The main limitation of the usual geometric flows without forcing term is that they tend to regularize too much the level sets and the asymptotic states are trivial. By including an appropriate forcing term in the equation we obtain more suitable models for level set regularization which can be useful for nonlinear filtering and image segmentation. 


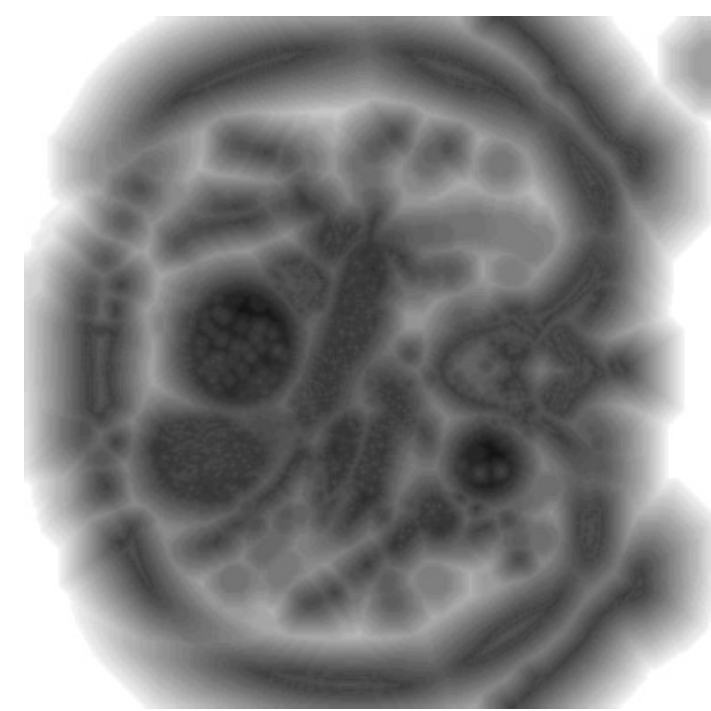

(a) signed distance $d_{U_{0}, \beta}(x)$ (video)

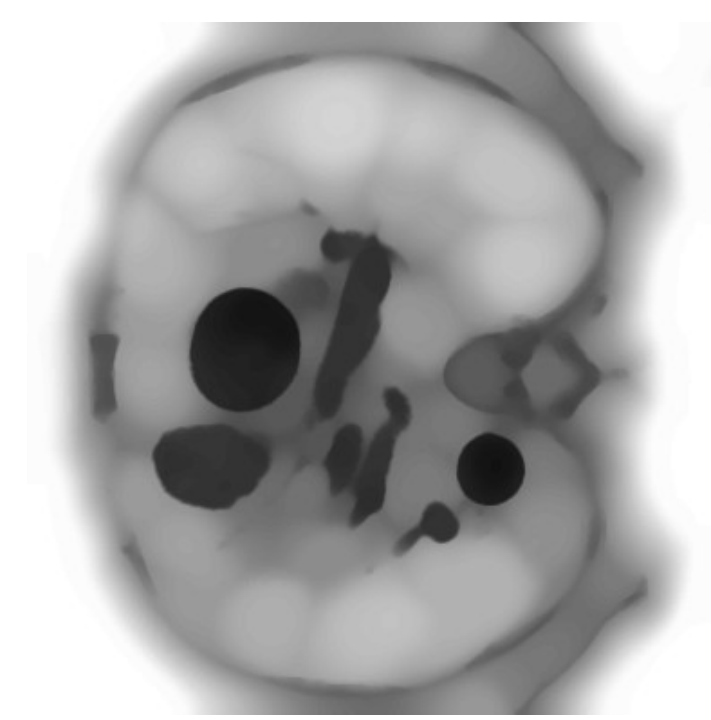

(c) $u(10, x)$ sol. (1)-(4) for $k_{0.1,0.2}^{3}(x)$ (video)

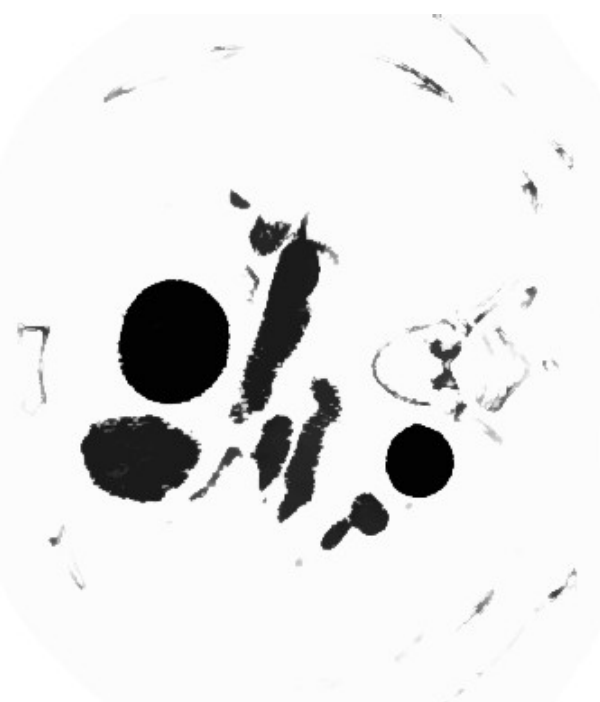

(b) $u(10, x)$ sol. of (1)-(4) for $k_{1 ., 0.2}^{3}(x)$ (video)

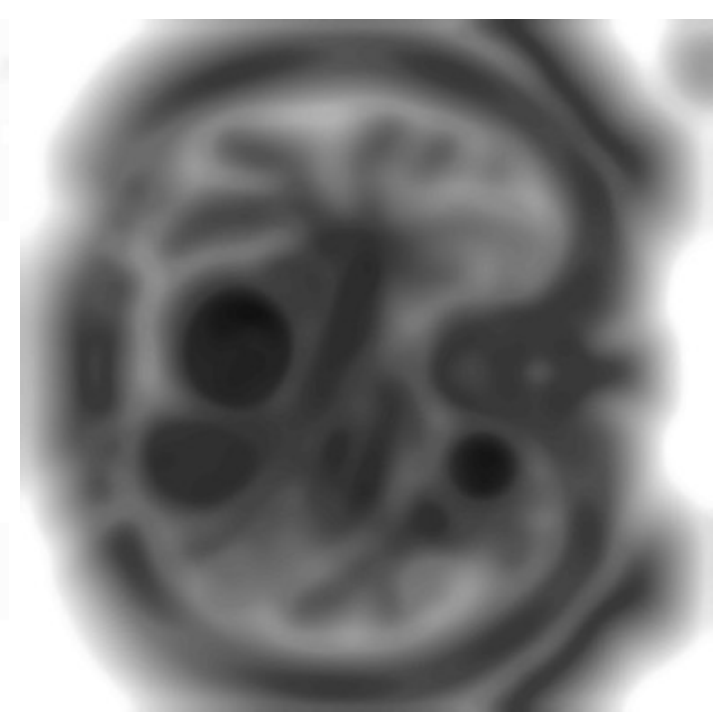

(d) $u(10, x)$ sol. (1)-(4) for $k(x) \equiv 0$ (video)

Figure 23. We show one slice of the signed distance function $d_{U_{0}, \beta}(x)$ as well as the solution of (1)-(4) for $t=0$ with different forcing terms. For each image we also include a link to a video with the full $3 D$ sequence.

In this paper we show some mathematical results on the solutions of these equations for a forcing term $k(x)$ being merely a bounded Hölder continuous function. We also studied in detail the shape of some radial solutions with nontrivial asymptotic states and we also present some results on the evolution of the level sets. We perform some experiments, using a basic explicit finite difference numerical scheme, to illustrate the level set regularization obtained with the proposed equations and the results are very promising. The level set is regularized by 
removing high curvature areas but at the same time it remains close to the original level set shape. As showed in the experiments, this behavior is quite useful in the context of nonlinear filtering or image segmentation. In particular, we show some interesting results on medical image segmentation.

\section{REFERENCES}

[1] L. Alvarez, C. Cuenca, J. Esclarín, L. Mazorra, and J.-M. Morel, Affine invariant distance using multiscale analysis, J. Math. Imaging Vision, 55 (2016), pp. 199-209.

[2] L. Alvarez, G. DíAz, And J. I. DíAz, Some qualitative properties for geometric flows and its Euler implicit discretization, Nonlinear Anal., 137 (2016), pp. 43-76.

[3] L. Alvarez, F. Guichard, P.-L. Lions, and J.-M. Morel, Axiomatisation et nouveaux opérateurs de la morphologie mathématique, C. R. Acad. Sci. Sér. 1 Math., 315 (1992), pp. 265-268.

[4] L. Alvarez, F. Guichard, P.-L. Lions, and J.-M. Morel, Axiomes et équations fondamentales du traitement d'images. (analyse multiéchelle et edp), C. R. Acad. Sci. Sér. 1 Math., 315 (1992), pp. $135-138$.

[5] L. Alvarez, F. Guichard, P.-L. Lions, and J.-M. Morel, Axioms and fundamental equations of image processing, Arch. Ration. Mech. Anal., 123 (1993), pp. 199-257.

[6] S. Angenent, Parabolic equations for curves on surfaces Part I. Curves with p-integrable curvature, Ann. of Math., 132 (1990), pp. 451-483.

[7] S. Angenent, G. Sapiro, and A. Tannenbaum, On the affine heat equation for non-convex curves, J. Amer. Math. Soc., 11 (1998), pp. 601-634.

[8] G. Aubert and P. Kornprobst, Mathematical Problems in Image Processing: Partial Differential Equations and the Calculus of Variations, 2nd ed., Springer, New York, 2010.

[9] M. Breuss And J. Weickert, Highly accurate schemes for PDE-based morphology with general convex structuring elements, Int. J. Comput. Vis., 92 (2011), pp. 132-145.

[10] V. Caselles, F. Catté, T. Coll, and F. Dibos, A geometric model for active contours in image processing, Numer. Math., 66 (1993), pp. 1-31.

[11] V. Caselles, B. Coll, And J.-M. Morel, Scale space versus topographic map for natural images, in Scale-Space Theory in Computer Vision, Lecture Notes in Comput. Sci. 1252, Springer, New York, 1997, pp. 29-49.

[12] V. Caselles, B. Coll, and J.-M. Morel, Topographic maps and local contrast changes in natural images, Int. J. Comput. Vis., 33 (1999), pp. 5-27.

[13] V. Caselles, R. Kimmel, And G. Sapiro, Geodesic active contours, Int. J. Comput. Vis., 22 (1997), pp. $61-79$.

[14] V. Caselles, R. Kimmel, G. Sapiro, and C. Sbert, Minimal surfaces: A geometric three dimensional segmentation approach, Numer. Math., 77 (1997), pp. 423-451.

[15] A. Cesaroni, M. Novaga, and E. Valdinoci, Curve shortening flow in heterogeneous media, Interfaces Free Bound., 13 (2011), pp. 485-505.

[16] Y. G. Chen, Y. Giga, AND S. Goto, Uniqueness and existence of viscosity solutions of generalized mean curvature flow equations, J. Differential Geom., 33 (1991), pp. 749-786.

[17] M. G. Crandall, H. Ishit, And P.-L. Lions, User's guide to viscosity solutions of second order partial differential equations, Bull. Amer. Math. Soc., 27 (1992), pp. 1-67.

[18] L. C. Evans And J. Spruck, Motion of level sets by mean curvature, J. Differential Geom., 33 (1991), pp. 635-681.

[19] N. Forcadel, Dislocation dynamics with a mean curvature term: Short time existence and uniqueness, Differential Integral Equations, 21 (2008), pp. 285-304.

[20] Y. Giga, Surface Evolution Equations: A Level Set Approach, Monogr. Math., Birkhäuser, Basel, 2006.

[21] Y. Giga, S. Goto, H. Ishi, AND M. H. Sato, Comparison principle and convexity preserving properties for singular degenerate parabolic equations on unbounded domains, Indiana Univ. Math. J., 40 (1991), pp. $443-470$.

[22] R. MANNIESING AND W. NIESSEN, Local speed functions in level set based vessel segmentation, in MICCAI 2004 Proceedings, Part I, Springer, Berlin, 2004, pp. 475-482. 
[23] G. Mercier, Curve-and-Surface Evolutions for Image Processing, Theses, École Polytechnique, 2015, https://pastel.archives-ouvertes.fr/.

[24] G. Mercier, Mean Curvature Flow with Obstacles: A Viscosity Approach, 2016. arXiv:1409.7657, 2016.

[25] G. Mercier And M. Novaga, Mean Curvature Flow with Obstacles: Existence, Uniqueness and Regularity of Solutions, arXiv:1409.7327, 2014.

[26] S. Osher And R. Fedkiw, Level Set Methods and Dynamic Implicit Surfaces, Appl. Math. Sci., 153, Springer, New York, 2002

[27] S. Osher AND J. A. Sethinan, Fronts propagating with curvature-dependent speed: Algorithms based on Hamilton-Jacobi formulations, J. Comput. Phys., 79 (1988), pp. 12-49.

[28] G. SAPIRo, Geometric Partial Differential Equations and Image Analysis, Cambridge University Press, Cambridge, UK, 2006.

[29] G. Sapiro and A. Tannenbaum, Affine invariant scale-space, Int. J. Comput. Vis., 11 (1993), pp. 25-44.

[30] J. A. Sethian, Level Set Methods and Fast Marching Methods, 2nd ed., Cambridge University Press, Cambridge, UK, 1999.

[31] J. Weickert And G. KüHne, Fast Methods for Implicit Active Contour Models, Geometric Level Set Methods in Imaging, Vision, and Graphics, Springer, New York, 2003, pp. 43-57. 